quatrième série-tome 46 fascicule 3 mai-juin 2013

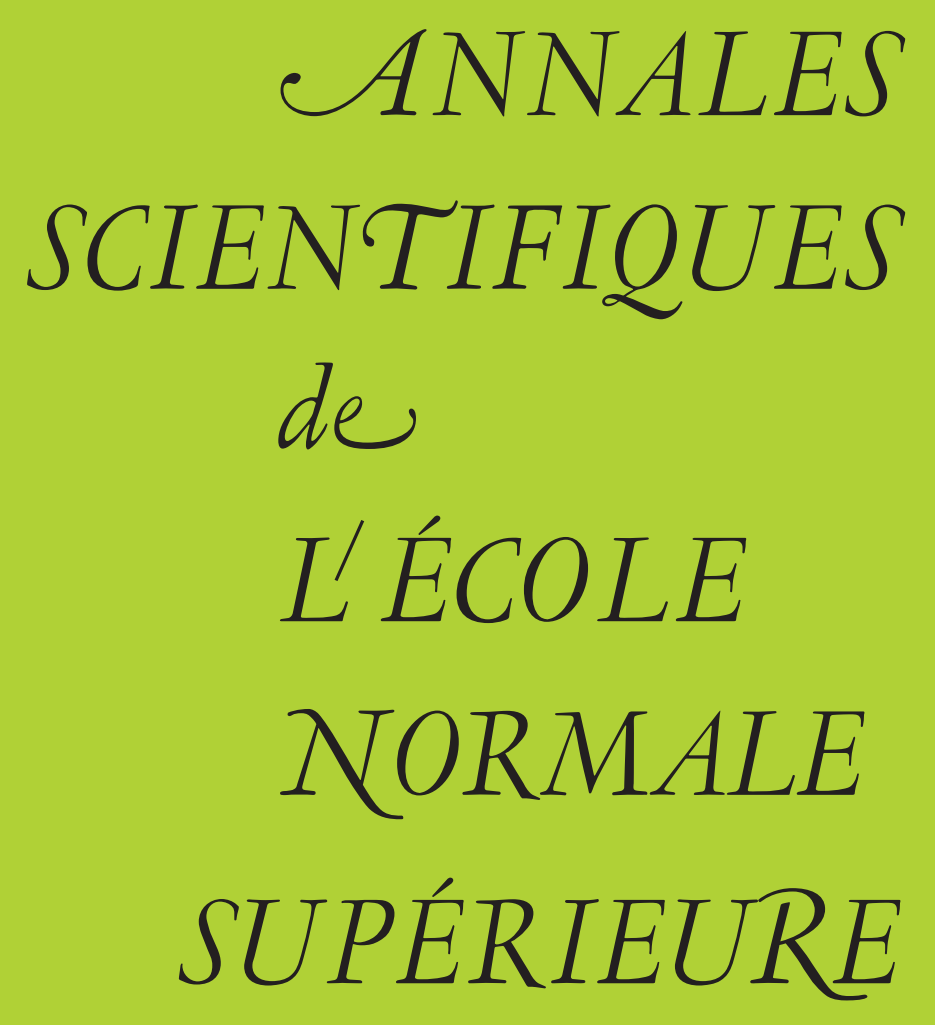

Baptiste CALMĖS \& Viktor PETROV \& Kirill ZAINOULLINE Imariants, torsion indices and oriented cohomology of complete flags 
Ann. Scient. Éc. Norm. Sup.

$4{ }^{\text {e }}$ série, t. 46, 2013, p. 405 à 448

\title{
INVARIANTS, TORSION INDICES AND ORIENTED COHOMOLOGY OF COMPLETE FLAGS
}

\author{
BY Baptiste CALMÈS, VikTor PETROV \\ AND Kirill ZAinOULLINE
}

\begin{abstract}
Let $G$ be a split semisimple linear algebraic group over a field and let $T$ be a split maximal torus of $G$. Let $\mathrm{h}$ be an oriented cohomology (algebraic cobordism, connective $K$-theory, Chow groups, Grothendieck's $K_{0}$, etc.) with formal group law $F$. We construct a ring from $F$ and the characters of $T$, that we call a formal group ring, and we define a characteristic ring morphism $c$ from this formal group ring to $\mathrm{h}(G / B)$ where $G / B$ is the variety of Borel subgroups of $G$. Our main result says that when the torsion index of $G$ is inverted, $c$ is surjective and its kernel is generated by elements invariant under the Weyl group of $G$. As an application, we provide an algorithm to compute the ring structure of $\mathrm{h}(G / B)$ and to describe the classes of desingularized Schubert varieties and their products.

RÉSumÉ. - Soit $G$ un groupe algébrique linéaire semi-simple déployé sur un corps et soit $T$ un tore maximal déployé de $G$. Étant donnée une cohomologie orientée h (anneau de Chow, $K_{0}$ de Grothendieck, $K$-théorie connective, etc.) et sa loi de groupe formel $F$, nous construisons un anneau appelé anneau de groupe formel, associé à $F$ et aux caractères de $T$, puis un homomorphisme caractéristique $c$ de cet anneau de groupe formel vers l'anneau $\mathrm{h}(G / B)$ où $G / B$ est la variété des sousgroupes de Borel de $G$. Le résultat principal de cet article montre que, lorsque l'indice de torsion du groupe $G$ est inversé, $c$ est surjectif et son noyau est engendré par des éléments invariants sous l'action du groupe de Weyl de $G$. En guise d'application, nous fournissons un algorithme qui permet de calculer la structure d'anneau de $\mathrm{h}(G / B)$ et d'y calculer les classes de variétés de Schubert désingularisées et leur produits.
\end{abstract}

\section{Introduction}

Let $\mathrm{H}$ be an algebraic cohomology theory endowed with Chern classes $c_{i}$ such that for any two line bundles $\mathscr{L}_{1}$ and $\mathscr{L}_{2}$ over a variety $X$ we have

$$
c_{1}\left(\mathscr{L}_{1} \otimes \mathscr{L}_{2}\right)=c_{1}\left(\mathscr{L}_{1}\right)+c_{2}\left(\mathscr{L}_{2}\right) .
$$

The first author was supported by EPSRC grant EP/E01786X/1. The second author was supported by RFBR 08-01-00756, 09-01-00878, 09-01-90304, 09-01-91333. The third author was supported by NSERC Discovery grants 385795 and 396100 . 
The basic example of such a theory is the Chow group $\mathrm{CH}$ of algebraic cycles modulo rational equivalence.

Let $G$ be a split semi-simple linear algebraic group over a field $k$ and let $T$ be a split maximal torus inside $G$ contained in a Borel subgroup $B$. Consider the variety $G / B$ of Borel subgroups of $G$ with respect to $T$. In two classical papers [7] and [8] Demazure studied the cohomology ring $\mathrm{H}(G / B ; \mathbb{Z})$ and provided an algorithm to compute $\mathrm{H}(G / B ; \mathbb{Z})$ in terms of generators and relations.

The main object of his consideration was the so called characteristic map

$$
\mathfrak{c}: S^{*}(M) \rightarrow \mathrm{H}(G / B ; \mathbb{Z}),
$$

where $S^{*}(M)$ is the symmetric algebra of the character group $M$ of $T$. In [7] Demazure interpreted this map from the point of view of invariant theory of the Weyl group $W$ of $G$ by identifying its kernel with the ideal generated by non-constant invariants $S^{*}(M)^{W}$. The cohomology ring $\mathrm{H}(G / B ; \mathbb{Z})$ was then replaced by a certain algebra constructed in terms of operators and defined in purely combinatorial terms.

In the present paper, we generalize most of the results of [7] to the case of an arbitrary oriented cohomology theory h, i.e., when (1) is replaced by

$$
c_{1}\left(\mathscr{L}_{1} \otimes \mathscr{L}_{2}\right)=F\left(c_{1}\left(\mathscr{L}_{1}\right), c_{1}\left(\mathscr{L}_{2}\right)\right)
$$

where $F$ is the formal group law associated to h. Such theories were extensively studied by Levine-Morel [14], Panin-Smirnov [16], Merkurjev [15] and others. Apart from the Chow ring, other examples include algebraic $K$-theory, étale cohomology $H_{\mathrm{et}}^{*}\left(-, \mu_{m}\right)$, $(m, \operatorname{char}(k))=1$, connective $K$-theory, elliptic cohomology and the universal such theory: algebraic cobordism $\Omega$.

To generalize the characteristic map (2), we first introduce a substitute for the symmetric algebra $S^{*}(M)=\mathrm{CH}^{*}(B T)$. This new combinatorial object, which we call a formal group ring, is denoted by $R \llbracket M \rrbracket_{F}$, where $R=\mathrm{h}(p t)$ is the coefficient ring, and can be viewed as a substitute of the cohomology ring of the classifying space $\mathrm{h}(B T)$ of $T$. In the case of a finite group $G$ this object is related to generalized group characters studied in [12]. As in [7], we introduce a subalgebra $\mathscr{D}(M)_{F}$ of the $R$-linear endomorphisms of $R \llbracket M \rrbracket_{F}$ generated by specific differential operators and by taking its $R$-dual we obtain $\mathcal{H}(M)_{F}$, a combinatorial substitute for the cohomology $\operatorname{ring} \mathrm{h}(G / B ; \mathbb{Z})$. The characteristic map (2) then turns into the map

$$
\mathfrak{c}: R \llbracket M \rrbracket_{F} \rightarrow \mathcal{H}(M)_{F} .
$$

The Weyl group $W$ acts naturally on $R \llbracket M \rrbracket_{F}$ and the main result of our paper (Theorems 6.4 and 6.9) says that:

THeOREM. - If the torsion index of $G$ is invertible in $R$ and $R$ has no 2-torsion, then the characteristic map is surjective and its kernel is generated by $W$-invariant elements in the augmentation ideal.

Demazure's methods to prove this theorem in the special case of the additive formal group law do not work in general, for the following reason: the main objects used in his proofs are operators $\Delta_{w}$ for every $w \in W$. They are defined first for simple reflections, and afterwards for any $w$ by decomposing it into simple reflections and composing the 
corresponding operators. It is then proved that the resulting composition is independent of the decomposition of $w$. For more general formal group laws, similar operators can still be defined for simple reflections (see Definition 3.5), but independence of the decomposition does not hold, as it was observed in [5]. Geometrically, it can be translated into the fact that the cobordism class of a desingularized Schubert variety depends on the desingularization, and not only on the Schubert variety itself (see Lemma 13.3). We overcome this problem by working with suitable filtrations such that the associated graded structures are covered by the additive case of Demazure (see in particular Proposition 4.4). We therefore encourage the reader unfamiliar with [7] to start by having a quick look at it before reading our Sections 3 to 7.

As an immediate application of the developed techniques, we provide an efficient algorithm for computing the cohomology ring $\mathcal{H}(M)_{F}=\mathrm{h}(G / B ; \mathbb{Z})$. To do this we generalize the Bott-Samelson approach introduced in [1] and [8]. For oriented topological theories, some algorithms were considered by Bressler-Evens in [4, 5] and for algebraic theories in characteristic 0 by Hornbostel-Kiritchenko in [13]. See Remark 15.2 for a comparison.

Note that the theorem also provides another approach to computing the cohomology ring $\mathrm{h}(G / B ; \mathbb{Z})$ by looking at the subring of invariants $R \llbracket M \rrbracket_{F}^{W}$. Observe that in the classical case when $\mathrm{h}=\mathrm{CH}$ (or $K_{0}$ ) and $G$ is simply-connected it is known that $R \llbracket M \rrbracket_{F}^{W}$ is a power series ring in basic polynomial invariants (resp. fundamental representations). In general, the structure of $R \llbracket M \rrbracket_{F}^{W}$ remains unknown.

Finally, for the reader primarily interested in topology, let us mention that while all our proofs are algebraic and written in the language of algebraic geometry, the results apply as they are to topological cobordism or other complex oriented theories. Indeed, there is a canonical ring morphism

$$
\Omega^{*}(G / B) \rightarrow \mathrm{MU}^{*}(G / B(\mathbb{C}))
$$

(see [14, Ex. 1.2.10]) that is an isomorphism because both are free modules over the Lazard ring with bases corresponding to each other (given by desingularized Schubert cells).

The paper consists of three parts.

In the first part, we generalize the results of [7] by introducing and studying the generalized characteristic map c : $R \llbracket M \rrbracket_{F} \rightarrow \mathcal{H}(M)_{F}$. In Section 2, we introduce the formal group ring $R \llbracket M \rrbracket_{F}$ and prove its main properties. In Section 3, we define the main operators $\Delta$ and $C$ on $R \llbracket M \rrbracket_{F}$. In Sections 4 and 5 we study the subalgebra $\mathscr{D}(M)_{F}$ of operators generated by $\Delta$ (resp. $C$ ) and multiplications. In Section 6 we define $\mathscr{H}(M)_{F}$ and prove the main theorem. In Section 7 we introduce a product on $\mathscr{H}(M)_{F}$ compatible with the characteristic map $\mathfrak{c}$.

In the second part, we generalize some of the results of [8] to arbitrary oriented cohomology theories. In Section 8, we discuss properties of oriented theories. In Sections 9 to 12, we carry out the Bott-Samelson desingularization approach.

In the last part, we apply the results of the first and the second parts to obtain information about the ring structure in $\mathrm{h}(G / B ; \mathbb{Z})$. In Section 13 , we prove that our algebraic replacement $\mathcal{H}(M)_{F}$ is isomorphic (as a ring) to the oriented cohomology $\mathrm{h}(G / B ; \mathbb{Z}$ ) and that the characteristic maps $\mathfrak{c}_{G / B}$ and $\mathfrak{c}$ correspond to each other via this isomorphism. In Section 14, we give an algebraic description of the push-forward to the point and we prove various 
formulas. In Sections 15 and 16, we explain an algorithm to compute the ring structure of $\mathrm{h}(G / B ; \mathbb{Z})$ and the Landweber-Novikov operations on algebraic cobordism. Finally, in Section 17 , we give multiplication tables for $\Omega^{*}(G / B ; \mathbb{Z})$ for groups $G$ of rank 2 .

\section{Notation}

Let $k$ denote a base field of arbitrary characteristic. A variety over $k$ means a reduced irreducible scheme of finite type over $k$. By $X$ and $Y$ we always mean smooth varieties over $k$. The base point Spec $k$ is denoted by pt.

A ring always means a commutative ring with a unit and $R$ always denotes a ring. A ring $R^{\prime}$ is called an $R$-algebra if it comes equipped with an injective ring homomorphism $R \hookrightarrow R^{\prime}$. The letter $M$ always denotes an Abelian group.

All formal group laws are assumed to be one-dimensional and commutative. Let $F$ denote a formal group law and let $\mathbb{L}$ denote the Lazard ring, i.e., the coefficient ring of the universal formal group law $U$.

\section{Acknowledgments}

Our sincere gratitude goes to Michel Demazure for his answers to our questions concerning the paper [7]. We would like to thank the authors of the Macaulay 2 software [10], and, in particular Dan Grayson for his quick and accurate responses on the Macaulay 2 mailing list. We would also like to thank Vladimir Chernousov, Fabien Morel and Burt Totaro for their encouraging attention to our work.

\section{PART I \\ INVARIANTS, TORSION INDICES AND FORMAL GROUP LAWS}

\section{Formal group rings}

Let $R$ be a ring, let $M$ be an Abelian group and let $F$ be a formal group law over $R$. In the present section we introduce and study the formal group ring $R \llbracket M \rrbracket_{F}$. For this, we use several auxiliary facts concerning topological rings and their completions which can be found in [3, III, §2]. The main result here is the decomposition Theorem 2.11. At the end we provide some examples of computations of $R \llbracket M \rrbracket_{F}$.

Definition 2.1. - Let $R$ be a ring and let $S$ be a set. Let $R\left[x_{S}\right]:=R\left[x_{s}, s \in S\right]$ denote the polynomial ring over $R$ with variables indexed by $S$. Let $\epsilon: R\left[x_{S}\right] \rightarrow R$ be the augmentation morphism which maps any $x_{s}$ to 0 . Consider the $\operatorname{ker}(\epsilon)$-adic topology on $R\left[x_{S}\right]$ given by ideals $\operatorname{ker}(\epsilon)^{i}, i \geq 0$, which form a fundamental system of open neighborhoods of 0 . Note that a polynomial is in $\operatorname{ker}(\epsilon)^{i}$ if and only if its valuation is at least $i$, hence, we have $\cap_{i} \operatorname{ker}(\epsilon)^{i}=\{0\}$ and the $\operatorname{ker}(\epsilon)$-adic topology is Hausdorff.

We define $R \llbracket x_{S} \rrbracket$ to be the $\operatorname{ker}(\epsilon)$-adic completion of the polynomial ring $R\left[x_{S}\right]$.

4 e SÉRIE - TOME $46-2013$ - No 3 
REMARK 2.2. - By definition, an element in $R \llbracket x_{S} \rrbracket$ can be written uniquely as a formal sum

$$
\sum_{s_{1}, \ldots, s_{m} \in S} a_{s_{1}, \ldots, s_{m}} \cdot x_{s_{1}} \ldots x_{s_{m}}, \quad a_{s_{1}, \ldots, s_{m}} \in R
$$

where for any positive $m$ there is only a finite number of non-zero coefficients $a_{s_{1}, \ldots, s_{m}}$. In particular, when $S$ is a finite set of order $n$, the ring $R \llbracket x_{S} \rrbracket$ is the usual ring of power series in $n$ variables.

2.3. - Let $F$ be a formal group law over a ring $R$ (see [14, p.4]). Given an integer $m \geq 0$ we use the notation

$$
x+_{F} y=F(x, y), \quad m \cdot \cdot_{F} x=\underbrace{x+{ }_{F} \ldots+_{F} x}_{m \text { times }} \text { and }(-m) \cdot_{F} x=-{ }_{F}\left(m \cdot{ }_{F} x\right) .
$$

By associativity of $F$, for any $m_{1}, m_{2} \in \mathbb{Z}$ we have

$$
\left(m_{1}+m_{2}\right) \cdot_{F} x=\left(m_{1} \cdot{ }_{F} x\right)+{ }_{F}\left(m_{2} \cdot{ }_{F} x\right) .
$$

Now, here comes the definition playing a central role in the sequel.

Definition 2.4. - Let $M$ be an Abelian group and let $F$ be a formal group law over a ring $R$. Consider the ring $R \llbracket x_{M} \rrbracket$ and let $g_{F}$ be the closure of the ideal generated by the elements $x_{0}$ and $x_{\lambda+\mu}-\left(x_{\lambda}+{ }_{F} x_{\mu}\right)$ for all $\lambda, \mu \in M$. We define the formal group ring $R \llbracket M \rrbracket_{F}$ to be the quotient

$$
R \llbracket M \rrbracket_{F}=R \llbracket x_{M} \rrbracket / \mathscr{\partial}_{F} .
$$

The class of $x_{\lambda}$ in $R \llbracket M \rrbracket_{F}$ will be denoted by the same letter.

2.5. - By definition, $R \llbracket x_{M} \rrbracket$ is a complete Hausdorff ring with respect to the topology induced by the kernel of the augmentation $R \llbracket x_{M} \rrbracket \rightarrow R$. Since $\mathcal{J}_{F}$ is clearly contained in this kernel, the augmentation map $R \llbracket x_{M} \rrbracket \rightarrow R$ factors through the quotient $R \llbracket M \rrbracket_{F}$. Therefore, $R \llbracket M \rrbracket_{F}$ is a complete Hausdorff ring with respect to the $\mathcal{J}_{F}$-adic topology, where $\mathcal{J}_{F}$ denotes the kernel of the augmentation $R \llbracket M \rrbracket_{F} \rightarrow R$.

Let $f: R \rightarrow R^{\prime}$ be a morphism of rings respecting the formal group laws, i.e., sending every coefficient of $F$ to the corresponding coefficient of $F^{\prime}$. Then, for every Abelian group $M, f$ induces a ring homomorphism $f_{\star}: R \llbracket M \rrbracket_{F} \rightarrow R^{\prime} \llbracket M \rrbracket_{F^{\prime}}$ sending $x_{\lambda} \in R \llbracket M \rrbracket_{F}$ to $x_{\lambda} \in R^{\prime} \llbracket M \rrbracket_{F^{\prime}}$ for every $\lambda \in M$. This morphism sends $\mathscr{I}_{F}$ to $\mathscr{I}_{F^{\prime}}$ and, hence, is continuous.

Let now $f: M \rightarrow M^{\prime}$ be a morphism of Abelian groups. It induces a continuous ring homomorphism $\hat{f}: R \llbracket M \rrbracket_{F} \rightarrow R \llbracket M^{\prime} \rrbracket_{F}$ sending $x_{\lambda}$ to $x_{f(\lambda)}$. Moreover, if $f$ is surjective, then so is $\hat{f}$.

Finally, let $f: F \rightarrow F^{\prime}$ be a morphism of formal group laws over $R$, i.e., a formal power series $f \in R \llbracket x \rrbracket$ such that $f\left(x+{ }_{F} y\right)=f(x)+{ }_{F^{\prime}} f(y)$. Then, $f$ induces a continuous ring homomorphism $f^{\star}: R \llbracket M \rrbracket_{F^{\prime}} \rightarrow R \llbracket M \rrbracket_{F}$ sending $x_{\lambda}$ to $f\left(x_{\lambda}\right)$.

We have therefore proved: 
Lemma 2.6. - Via the above constructions $(-)_{\star},(\hat{-})$ and $(-)^{\star}$, the assignment taking $(R, M, F)$ to the topological ring $R \llbracket M \rrbracket_{F}$ is covariant with respect to ring morphisms $R \rightarrow R^{\prime}$ and morphisms of Abelian groups $M \rightarrow M^{\prime}$, and is contravariant with respect to morphisms of formal group laws $F \rightarrow F^{\prime}$.

2.7. - Let $A$ be an $R$-algebra with an ideal $J$ such that $A$ is Hausdorff and complete for the $I$-adic topology. By scalar extension, we may consider the formal group law $F$ as an element of $A \llbracket x, y \rrbracket$. Let $a, b \in \mathcal{J}$. The specialization at $x=a$ and $y=b$ defines a pairing

$$
\boxplus: I \times I \rightarrow I .
$$

Similarly, we define $\boxminus: I \rightarrow I$ using the inverse of $F$ and $\square: \mathbb{Z} \times \mathscr{I} \rightarrow \mathscr{I}$ by applying $\boxplus$ or $\boxminus$ iteratively. From the associativity, commutativity and inverse properties of $F$, one shows that $(\mathcal{I}, \boxplus)$ is an Abelian group, in which the inverse of $a \in \mathcal{I}$ is $\boxminus a$. In particular, when $A=R \llbracket M \rrbracket_{F}$ and $\mathscr{J}=\mathcal{I}_{F}$, from the continuity of the quotient map $R \llbracket x_{M} \rrbracket \rightarrow R \llbracket M \rrbracket_{F}$ it follows that $x_{\lambda+\mu}=x_{\lambda} \boxplus x_{\mu}$ and

$$
x_{-\lambda}=x_{-\lambda} \boxplus\left(x_{\lambda} \boxminus x_{\lambda}\right)=\left(x_{-\lambda} \boxplus x_{\lambda}\right) \boxminus x_{\lambda}=0 \boxminus\left(x_{\lambda}\right)=\boxminus\left(x_{\lambda}\right) .
$$

Therefore $x: \lambda \mapsto x_{\lambda}$ is a group homomorphism from $M$ to $\left(\mathcal{J}_{F}, \boxplus\right)$.

2.8. - We consider the category of triples $(A, I, f)$ where $I$ is an ideal of $A$, a complete Hausdorff $R$-algebra for the $I$-adic topology, and $f$ is a group homomorphism from $M$ to $(I, \boxplus)$. The morphisms from an object $(A, I, f)$ to an object $\left(A^{\prime}, I^{\prime}, f^{\prime}\right)$ are continuous $R$-algebra morphisms $\phi: A \rightarrow A^{\prime}$ such that $\phi \circ f=f^{\prime}$. It is straightforward that $\left(R \llbracket M \rrbracket_{F}, \searrow_{F}, x\right)$ is an initial object in this category, and is thus uniquely defined (up to unique isomorphism) by that universal property. ${ }^{(1)}$

2.9. - Let $f: R \rightarrow R^{\prime}$ be a morphism of rings. The morphism $f_{\star}$ of Lemma 2.6 induces a morphism of $R$-algebras

$$
R^{\prime} \otimes_{R} R \llbracket M \rrbracket_{F} \rightarrow R^{\prime} \llbracket M \rrbracket_{F}, \quad r^{\prime} \otimes z \mapsto r^{\prime} \cdot f_{\star}(z)
$$

sending $R^{\prime} \otimes \mathscr{J}_{F}$ to $\mathscr{J}_{F}^{\prime}$, kernel of the augmentation map on $R^{\prime} \llbracket M \rrbracket_{F}$. It is thus continuous for the corresponding adic topologies and therefore extends to a morphism between completed rings

$$
\left(R^{\prime} \otimes_{R} R \llbracket M \rrbracket_{F}\right)^{\wedge} \rightarrow R^{\prime} \llbracket M \rrbracket_{F}
$$

( $R \llbracket M \rrbracket_{F}$ is already complete). This morphism is an isomorphism; the universal property 2.8 of $R^{\prime} \llbracket M \rrbracket_{F}$ gives the inverse morphism. In particular, let $\mathbb{L}$ be the Lazard ring of coefficients of the universal formal group law $U$. For any formal group law $F$ over $R$, there is a unique ring homomorphism $\vartheta_{F}: \mathbb{L} \rightarrow R$ sending the universal formal group law $U$ on $\mathbb{L}$ to $F$. So

$$
\left(R \otimes_{\mathbb{L}} \mathbb{L} \llbracket M \rrbracket_{U}\right)^{\wedge} \simeq R \llbracket M \rrbracket_{F} .
$$

2.10. - A formal group law $F$ on $R$ induces via a ring homomorphism $R \rightarrow R^{\prime}$ a formal group law on $R^{\prime}$. In particular, if $M$ and $N$ are Abelian groups, then using $R \rightarrow R \llbracket M \rrbracket_{F}=R^{\prime}$,

(1) The universal property was first made explicit in [18, Def. 3.1.2], following the first version of the present paper. 
we can define $R \llbracket M \rrbracket_{F} \llbracket N \rrbracket_{F}$, which is naturally an $R \llbracket M \rrbracket_{F}$-algebra. By functoriality, $R \llbracket M \oplus N \rrbracket_{F}$ is also an $R \llbracket M \rrbracket_{F}$-algebra. We define two morphisms of $R \llbracket M \rrbracket_{F}$-algebras

$$
\phi: R \llbracket M \oplus N \rrbracket_{F} \rightarrow R \llbracket M \rrbracket_{F} \llbracket N \rrbracket_{F} \text { and } \psi: R \llbracket M \rrbracket_{F} \llbracket N \rrbracket_{F} \rightarrow R \llbracket M \oplus N \rrbracket_{F}
$$

as follows:

The map $R\left[x_{M \oplus N}\right] \rightarrow R \llbracket x_{M} \rrbracket \llbracket y_{N} \rrbracket$ sending $x_{(\lambda, \gamma)}$ to $x_{\lambda}+{ }_{F} y_{\gamma}$ extends to a continuous map $f: R \llbracket x_{M \oplus N} \rrbracket \rightarrow R \llbracket x_{M} \rrbracket \llbracket y_{N} \rrbracket$. Consider the composition

$$
R \llbracket x_{M \oplus N} \rrbracket \stackrel{f}{\rightarrow} R \llbracket x_{M} \rrbracket \llbracket y_{N} \rrbracket \stackrel{g}{\rightarrow} R \llbracket M \rrbracket_{F} \llbracket y_{N} \rrbracket \stackrel{h}{\rightarrow} R \llbracket M \rrbracket_{F} \llbracket N \rrbracket_{F}
$$

where $g$ is induced by $R \llbracket x_{M} \rrbracket \rightarrow R \llbracket M \rrbracket_{F}$ and $h$ is the quotient map. We have

$$
\begin{aligned}
h \circ g \circ f & \left(x_{(\lambda, \gamma)}+{ }_{F} x_{(\mu, \delta)}\right) \stackrel{(1)}{=} h \circ g\left(\left(x_{\lambda}+_{F} y_{\gamma}\right)+_{F}\left(x_{\mu}+{ }_{F} y_{\delta}\right)\right) \\
& \stackrel{(2)}{=} h \circ g\left(\left(x_{\lambda}+_{F} x_{\mu}\right)+_{F}\left(y_{\gamma}+_{F} y_{\delta}\right)\right) \stackrel{(3)}{=} h\left(\left(x_{\lambda} \boxplus x_{\mu}\right)+_{F}\left(y_{\gamma}+_{F} y_{\delta}\right)\right) \\
& \stackrel{(4)}{=}\left(x_{\lambda} \boxplus x_{\mu}\right)+_{F}\left(y_{\gamma} \boxplus y_{\delta}\right) \stackrel{(5)}{=} x_{\lambda+\mu}+_{F} y_{\gamma+\delta} \stackrel{(6)}{=} h\left(x_{\lambda+\mu}+_{F} y_{\gamma+\delta}\right) \\
& \stackrel{(7)}{=} h \circ g\left(x_{\lambda+\mu}+_{F} y_{\gamma+\delta}\right) \stackrel{(8)}{=} h \circ g \circ f\left(x_{(\lambda+\mu, \gamma+\delta)}\right),
\end{aligned}
$$

where (1) and (8) hold by definition and continuity of $f,(2)$ by associativity and commutativity of $F$, (3) and (7) by definition of $g$, (4) and (6) by definition of $h$ and (5) by definition of $\boxplus$. It shows that $h \circ g \circ f$ factors through $R \llbracket M \oplus N \rrbracket_{F}$ as

$$
h \circ g \circ f: R \llbracket x_{M \oplus N} \rrbracket \rightarrow R \llbracket M \oplus N \rrbracket_{F} \stackrel{\phi}{\rightarrow} R \llbracket M \rrbracket_{F} \llbracket N \rrbracket_{F},
$$

where $\phi$ is a morphism of $R \llbracket M \rrbracket_{F}$-algebras.

In the opposite direction, we proceed as follows: By functoriality, $R \llbracket M \oplus N \rrbracket_{F}$ is an $R \llbracket M \rrbracket_{F}$-algebra and it has an augmentation map back to $R \llbracket M \rrbracket_{F}$, whose kernel is temporarily denoted by $\tilde{I}_{F}$. It is complete for the $\tilde{\mathscr{J}}_{F}$-adic topology. The group homomorphism $N \rightarrow\left(\tilde{\mathscr{I}}_{F}, \boxplus\right)$ sending $\lambda$ to $x_{(0, \lambda)}$ therefore gives, by the universal property 2.8 , a continuous morphism of $R \llbracket M \rrbracket_{F}$-algebras $\psi: R \llbracket M \rrbracket_{F} \llbracket N \rrbracket_{F} \rightarrow R \llbracket M \oplus N \rrbracket_{F}$. We have $\psi\left(x_{\lambda}\right)=x_{(\lambda, 0)}$ for $\lambda \in M$ because $\psi$ is a morphism of $R \llbracket M \rrbracket_{F}$-algebras and $\psi\left(y_{\mu}\right)=x_{(0, \mu)}$ for $\mu \in N$ by the universal property.

THEOREM 2.11. - The morphisms of $R \llbracket M \rrbracket_{F}$-algebras $\phi$ and $\psi$ defined above are inverses to each other. In other words, we have a natural isomorphism

$$
R \llbracket M \oplus N \rrbracket_{F} \simeq R \llbracket M \rrbracket_{F} \llbracket N \rrbracket_{F} .
$$

Proof. - We have

$$
\psi \circ \phi\left(x_{(\lambda, \gamma)}\right)=\psi\left(x_{\lambda}+{ }_{F} y_{\gamma}\right)=\psi\left(x_{\lambda}\right)+{ }_{F} \psi\left(y_{\gamma}\right)=x_{(\lambda, 0)}+{ }_{F} x_{(0, \gamma)}=x_{(\lambda, \gamma)}
$$

where the second equality holds by continuity of $\psi$. The other composition can be checked on $y_{\gamma}$, since we are dealing with morphisms of $R \llbracket M \rrbracket_{F}$-algebras, and we have $\phi \circ \psi\left(y_{\gamma}\right)=$ $\phi\left(x_{(0, \gamma)}\right)=x_{0}+{ }_{F} y_{\gamma}=0+{ }_{F} y_{\gamma}=y_{\gamma}$.

Lemma 2.12. - Let $M=\mathbb{Z}$. Then sending $x_{m}$ to $m \cdot{ }_{F} x$ defines an isomorphism of $R$-algebras

$$
R \llbracket \mathbb{Z} \rrbracket_{F} \simeq R \llbracket x \rrbracket .
$$

In particular, if $M$ is a free Abelian group of rank one, then $R \llbracket M \rrbracket_{F}$ is isomorphic to $R \llbracket x \rrbracket$. 
Proof. - By the universal property 2.8, the group homomorphism $\mathbb{Z} \rightarrow \operatorname{ker}(\epsilon)$ sending $n$ to $n \square x$ yields a morphism of $R$-algebras $\phi: R \llbracket \mathbb{Z} \rrbracket_{F} \rightarrow R \llbracket x \rrbracket$. A map $\psi$ in the opposite direction is defined similarly using completeness, by sending $x$ to $x_{1}$. By continuity, checking that $\phi \circ \psi=$ id and $\psi \circ \phi=$ id can be done on generators. Namely, $\phi \circ \psi(x)=\phi\left(x_{1}\right)=x$ by definition, and $\psi \circ \phi\left(x_{n}\right)=\psi(n \boxminus x)=n \boxminus \psi(x)=n \boxminus x_{1}=x_{n}$.

Corollary 2.13. - Let $\phi: M \rightarrow \mathbb{Z}^{\oplus n}$ be an isomorphism. Then $\phi$ induces an isomorphism of $R$-algebras

$$
R \llbracket M \rrbracket_{F} \simeq R \llbracket x_{1}, \ldots, x_{n} \rrbracket
$$

Proof. - It follows from Lemma 2.12 and Theorem 2.11 by induction on $n$.

REMARK 2.14. - Note that the right hand side is independent of $F$, although the isomorphism depends on $F$. Also note that if $R$ is an integral domain, so is $R \llbracket \mathbb{Z}^{\oplus n} \rrbracket_{F}$.

EXAmple 2.15. - Similarly, one can prove that $R \llbracket \mathbb{Z} / n \rrbracket_{F} \simeq R \llbracket x \rrbracket /\left(n \cdot_{F} x\right)$ by sending $x_{m}$ to $m \cdot{ }_{F} x$ (cf. $[12, \S 6]$ for $\left.n=p^{r}\right)$. Observe that $n \cdot{ }_{F} x=n x+x^{2} q$. In particular, if $n$ is invertible in $R$, then $\left(n \cdot_{F} x\right)=(x)$ and $R \llbracket \mathbb{Z} / n \rrbracket_{F} \simeq R$.

Let us now examine what happens at a finite level in $R \llbracket M \rrbracket_{F}$.

2.16. - Let $R[M]_{F}$ denote the subring of $R \llbracket M \rrbracket_{F}$ defined as the image of the subring $R\left[x_{M}\right]$ by means of the composition $R\left[x_{M}\right] \rightarrow R \llbracket x_{M} \rrbracket \rightarrow R \llbracket M \rrbracket_{F}$. Then the $\operatorname{ring} R \llbracket M \rrbracket_{F}$ is the completion of $R[M]_{F}$ at the ideal $\mathscr{J}_{F} \cap R[M]_{F}$. By the functoriality of $R \llbracket M \rrbracket_{F}$ (see 2.6) the assignment

$$
(R, M, F) \mapsto R[M]_{F}
$$

is a covariant functor with respect to morphism of rings $R \rightarrow R^{\prime}$ and morphisms of Abelian groups $M \rightarrow M^{\prime}$. Moreover, if $M \rightarrow M^{\prime}$ is surjective, then so is $R[M]_{F} \rightarrow R\left[M^{\prime}\right]_{F}$.

EXAmple 2.17. - If $M=\mathbb{Z}$, the isomorphism of Lemma 2.12 maps $R[\mathbb{Z}]_{F}$ to polynomials in $n \cdot{ }_{F} x$. In particular, unless $F$ and the formal inverse have a finite number of nonzero coefficients only, $R[\mathbb{Z}]_{F}$ does not map to $R[x]$.

REMARK 2.18. - The morphism $\phi$ from 2.10 does not necessarily send $R[M \oplus N]_{F}$ to $R[M]_{F}[N]_{F}$. The morphism $\psi$ from 2.10 sends $R[M]_{F}[N]_{F}$ into $R[M \oplus N]_{F}$ but is not necessarily surjective.

ExAmple 2.19 (cf. 8.3). - The additive formal group law over $R$ is given by $F(x, y)=x+y$. In this case we have ring isomorphisms

$$
R \llbracket M \rrbracket_{F} \simeq \prod_{i=0}^{\infty} S_{R}^{i}(M) \text { and } R[M]_{F} \simeq \bigoplus_{i=0}^{\infty} S_{R}^{i}(M)
$$

where $S_{R}^{i}(M)$ is the $i$-th symmetric power of $M$ over $R$ and the isomorphisms are induced by sending $x_{\lambda}$ to $\lambda \in S_{R}^{1}(M)$.

4 e SÉRIE - TOME 46 - 2013 - No 3 
EXAMPle 2.20 (cf. 8.4). - The multiplicative periodic formal group law over $R$ is given by $F(x, y)=x+y-\beta x y$, where $\beta$ is an invertible element in $R$. Consider the group ring

$$
R[M]:=\left\{\sum_{j} r_{j} e^{\lambda_{j}} \mid r_{j} \in R, \lambda_{j} \in M\right\} .
$$

Let $\operatorname{tr}: R[M] \rightarrow R$ be the trace map, i.e., a $R$-linear map sending any $e^{\lambda}$ to 1 . Let $R[M]^{\wedge}$ be the completion of $R[M]$ at $\operatorname{ker}(\operatorname{tr})$. Then we have ring isomorphisms

$$
R \llbracket M \rrbracket_{F} \simeq R[M]^{\wedge} \text { and } R[M]_{F} \simeq R[M]
$$

induced by $x_{\lambda} \mapsto \beta^{-1}\left(1-e^{\lambda}\right)$ and $e^{\lambda} \mapsto\left(1-\beta x_{\lambda}\right)=\left(1-\beta x_{-\lambda}\right)^{-1}$.

EXAMPle 2.21 (cf. 8.5). - The multiplicative non-periodic formal group law over $R$ is given by $F(x, y)=x+y-v x y$, where $v$ is not invertible in $R$. Specializing $R \llbracket M \rrbracket_{F}$ at $v=0$ or $v=\beta$ where $\beta$ is invertible in $R$, we obtain the formal group rings of the previous examples.

REMARK 2.22. - Let $h$ be an oriented cohomology theory as defined in [14, Def. 1.1.2] and let $F$ be the associated formal group law (see Section 8). Let $M$ be the group of characters of a split torus $T$ over $k$. Then the formal group ring $R \llbracket M \rrbracket_{F}$ can be viewed as an algebraic substitute of the equivariant cohomology $\operatorname{ring} \mathrm{h}_{T}(\mathrm{pt})$ or of the cohomology ring of the classifying space $\mathrm{h}(B T)$.

\section{Differential operators $\Delta_{\alpha}$ and $C_{\alpha}$}

In the present section we introduce two linear operators on $R \llbracket M \rrbracket_{F}$. The first operator $\Delta_{\alpha}^{F}$ is a generalized version of the operator $\Delta_{\alpha}$ from [7, $\$ 3$ and $\S 4$ ]. Indeed, one recovers the results of loc. cit. when $R=\mathbb{Z}$ and $F$ is the additive formal group law from Example 2.19. A version of the second operator $C_{\alpha}^{F}$ was already used in $[5, \S 5]$ for topological complex cobordism.

3.1. - Consider a reduced root datum ${ }^{(2)}$ as in [7, $\left.\$ 1\right]$, i.e., a free $\mathbb{Z}$-module $M$ of finite rank, a finite subset of $M$ whose elements are called roots and a map associating a coroot $\alpha^{\vee} \in M^{\vee}$ to a root $\alpha$, satisfying certain axioms. The reflection map $\lambda \mapsto \lambda-\alpha^{\vee}(\lambda) \alpha$ is denoted by $s_{\alpha}$.

3.2. - The Weyl group $W$ associated to a reduced root datum is the subgroup of linear automorphisms of $M$ generated by reflections $s_{\alpha}$. It acts linearly on $M$ and thus by $R$-algebra automorphisms on $R \llbracket M \rrbracket_{F}$ using the functoriality in $M$ of $R \llbracket M \rrbracket_{F}$. We have the following obvious equalities for any $u \in R \llbracket M \rrbracket_{F}$ :

$$
s_{\alpha}(u)=s_{-\alpha}(u) \quad \text { and } \quad s_{\alpha}^{2}(u)=u .
$$

Lemma 3.3. - For any $n \in \mathbb{Z}$, the element $x-x+{ }_{F}\left(n \cdot{ }_{F} y\right)$ is uniquely divisible by $y$ in $R \llbracket x, y \rrbracket$.

Proof. - Since $y$ is a regular element in $R \llbracket x, y \rrbracket$, we just need to prove divisibility. Note that for any power series $g(x, y)$, the series $g(x, 0)-g(x, y)$ is divisible by $y$. Apply it to $g(x, y)=x+{ }_{F}\left(n \cdot{ }_{F} y\right)$.

(2) In [7], this is called a "système de racines précisé". 
Corollary 3.4. - Assume that $R$ is integral. For any $u \in R \llbracket M \rrbracket_{F}$, the element $u-s_{\alpha}(u)$ is uniquely divisible by $x_{\alpha}$.

Proof. - First note that $R \llbracket M \rrbracket_{F}$ is an integral domain by 2.14, so we just need to prove divisibility. Since

$$
s_{\alpha}\left(x_{\lambda}\right)=x_{\lambda-\alpha^{\vee}(\lambda) \alpha}=x_{\lambda} \boxplus\left(-\alpha^{\vee}(\lambda) \square x_{\alpha}\right)
$$

it holds by the previous lemma when $u=x_{\lambda}$. Then, by the formula

$$
u v-s_{\alpha}(u v)=\left(u-s_{\alpha}(u)\right) v+u\left(v-s_{\alpha}(v)\right)-\left(u-s_{\alpha}(u)\right)\left(v-s_{\alpha}(v)\right)
$$

the result holds by induction on the degree of monomials for any element in $R[M]_{F}$. Finally, it holds by density on the whole $R \llbracket M \rrbracket_{F}$.

For a given root $\alpha$ we define a linear operator $\Delta_{\alpha}^{F}$ on $R \llbracket M \rrbracket_{F}$ as follows:

Definition 3.5. - First, using Corollary 3.4 we define a linear operator $\Delta_{\alpha}^{U}$ on $\mathbb{L} \llbracket M \rrbracket_{U}$, where $U$ is the universal formal group law over the Lazard ring $\mathbb{L}$, as

$$
\Delta_{\alpha}^{U}(u)=\frac{u-s_{\alpha}(u)}{x_{\alpha}}, \text { where } u \in \mathbb{L} \llbracket M \rrbracket_{U} .
$$

Finally, identifying $R \llbracket M \rrbracket_{F}$ with $\left(R \otimes_{\mathbb{L}} \mathbb{L} \llbracket M \rrbracket_{U}\right)^{\wedge}$ via the isomorphism from 2.9 we define the desired operator $\Delta_{\alpha}^{F}$ on $R \llbracket M \rrbracket_{F}$ as the extension of the continuous operator $\operatorname{id}_{R} \otimes \Delta_{\alpha}^{U}$. We simply write $\Delta_{\alpha}(u)$ when the formal group law $F$ over $R$ is understood.

REMARK 3.6. - Observe that if $R$ is integral, then the operator $\Delta^{F}$ can be defined directly using the same formula as for $\Delta^{U}$. If $R$ is torsion, then it is not the case. Indeed, take $R=\mathbb{Z} / 2, M=\mathbb{Z}, F$ to be an additive law and $\alpha=2$ to be a root. Then $x_{\alpha}=x_{1+1}=$ $x_{1}+x_{1}=2 x_{1}=0$ in $R \llbracket M \rrbracket_{F}$.

REMARK 3.7. - We could instead have defined $\Delta_{\alpha}$ as $\Delta_{\alpha}(u)=\frac{s_{\alpha}(u)-u}{x_{-\alpha}}$. This exchanges $\Delta_{\alpha}$ with $-\Delta_{-\alpha}$ so it is easy to switch from one convention to the other. Both conventions give the same classical operator when the formal group law is additive.

Proposition 3.8. - The following formulas hold for any $u, v \in R \llbracket M \rrbracket_{F}, \lambda \in M$ and $w \in W$ (Compare to $\left.[7, \S 3]^{(3)}\right)$.

1. $\Delta_{\alpha}(1)=0, \quad \Delta_{\alpha}(u) x_{\alpha}=u-s_{\alpha}(u)$,

2. $\Delta_{\alpha}^{2}(u) x_{\alpha}=\Delta_{\alpha}(u)+\Delta_{-\alpha}(u), \quad \Delta_{\alpha}(u) x_{\alpha}=\Delta_{-\alpha}(u) x_{-\alpha}$,

3. $s_{\alpha} \Delta_{\alpha}(u)=-\Delta_{-\alpha}(u), \quad \Delta_{\alpha} s_{\alpha}(u)=-\Delta_{\alpha}(u)$,

4. $\Delta_{\alpha}(u v)=\Delta_{\alpha}(u) v+u \Delta_{\alpha}(v)-\Delta_{\alpha}(u) \Delta_{\alpha}(v) x_{\alpha}=\Delta_{\alpha}(u) v+s_{\alpha}(u) \Delta_{\alpha}(v)$,

5. $w \Delta_{\alpha} w^{-1}(u)=\Delta_{w(\alpha)}(u)$.

Proof. - All formulas can be proved in $\mathbb{L} \llbracket M \rrbracket_{U}$ (which is an integral domain) and then specialized to any other $R \llbracket M \rrbracket_{F}$. Formula (4) follows from Equation (3) above. Formula (5) follows from the fact that $\alpha^{\vee}\left(w^{-1}(\lambda)\right)=w(\alpha)^{\vee}(\lambda)$. All other formulas follow by definition.

(3) There is a sign mistake in Equation (3) of [7, §3]. One should read $\Delta_{\alpha}=-\Delta_{-\alpha}$ 
From now on, we fix a basis of simple roots $\left(\alpha_{1}, \ldots, \alpha_{n}\right)$, with associated simple reflections $s_{1}, \ldots, s_{n}$. This defines a length function on $W$. Let $I$ denote a sequence $\left(i_{1}, \ldots, i_{l}\right)$ of $l$ integers in $[1, n]$ and let $w(I)=s_{i_{1}} \cdots s_{i_{l}}$ be the corresponding product of simple reflections. The decomposition $I$ is reduced if $w$ has length $l$.

Definition 3.9. - The linear operator $\Delta_{I}^{F}$ is defined as the composition

$$
\Delta_{I}^{F}=\Delta_{\alpha_{i_{1}}}^{F} \circ \cdots \circ \Delta_{\alpha_{i_{l}}^{F}} .
$$

As usual, we write $\Delta_{I}$ for $\Delta_{I}^{F}$ when $F$ is understood.

The operators $\Delta_{I}$ have the following important property:

THEOREM 3.10. - Let $F$ be a formal group law of the form $F(x, y)=x+y-v \cdot x y$ for some $v \in R$. Let $I$ and $I^{\prime}$ be two reduced decompositions of $w$ in simple reflections. Then $\Delta_{I}=\Delta_{I^{\prime}}$.

Proof. - See [8, Theorem 2 p. 86]. The proof assumes $v=1$ but it works for any other value.

When the formal group law is of the above type, the previous theorem justifies the notation $\Delta_{w}$ instead of $\Delta_{I}$, but in general, we have to keep the dependence on the decomposition.

Remark 3.11. - Theorem 3.10 was proved in [4] (see loc. cit. Thm. 3.7) for topological oriented theories. Moreover, the result of [4] says that the formal group law has to be of the above form for such an independence of the decomposition to hold.

For a given root $\alpha$ we define another linear operator $C_{\alpha}^{F}$ on $R \llbracket M \rrbracket_{F}$ as follows:

Definition 3.12. - Let $g(x, y)$ be the power series defined by

$$
x+{ }_{F} y=x+y-x y \cdot g(x, y)
$$

and let $e_{\alpha}$ be the element $g\left(x_{\alpha}, x_{-\alpha}\right)$ in $R \llbracket M \rrbracket_{F}$. We set

$$
C_{\alpha}^{F}(u)=u e_{\alpha}-\Delta_{\alpha}^{F}(u), \text { where } u \in R \llbracket M \rrbracket_{F} .
$$

The geometric meaning of the operator $C_{\alpha}^{F}$ will be explained in 10.10. Note that there is no difference between $C_{\alpha}^{F}$ and $\Delta_{\alpha}^{F}$ when $e_{\alpha}=0$, i.e., when $F$ is additive. For a sequence of integers $I$ in $[1, n]$, we define the operator $C_{I}^{F}$ in the same way as $\Delta_{I}^{F}$. Again, we simply write $C_{\alpha}$ or $C_{I}$ when the formal group law $F$ is clear from the context.

Proposition 3.13. - The following formulas hold for any $u, v \in R \llbracket M \rrbracket_{F}, \lambda \in M$ and $w \in W$.

1. $C_{\alpha}(1)=e_{\alpha}, \quad C_{\alpha}\left(x_{-\alpha}\right)=2$,

2. $C_{\alpha}(u) x_{\alpha} x_{-\alpha}=u x_{\alpha}+s_{\alpha}(u) x_{-\alpha}, \quad C_{\alpha}\left(u x_{-\alpha}\right)=u+s_{\alpha}(u)$,

3. $C_{\alpha} s_{\alpha}(u)=C_{-\alpha}(u), \quad s_{\alpha} C_{\alpha}(u)=C_{\alpha}(u)$,

4. $C_{\alpha}(u v)=C_{\alpha}(u) v+s_{\alpha}(u) C_{\alpha}(v)-s_{\alpha}(u) v e_{\alpha}=C_{\alpha}(u) v-s_{\alpha}(u) \Delta_{\alpha}(v)$,

5. $w C_{\alpha} w^{-1}(u)=C_{w(\alpha)}(u)$,

6. $C_{\alpha} \Delta_{\alpha}=\Delta_{\alpha} C_{\alpha}=\Delta_{\alpha} C_{-\alpha}=0$.

Proof. - All these formulas can easily be derived from the ones of Proposition 3.8 using the definition of $C_{\alpha}$ and the fact that $e_{\alpha} x_{\alpha} x_{-\alpha}=x_{\alpha}+x_{-\alpha}$. 
Proposition 3.14. - Let $W_{\alpha}$ be the subgroup of order 2 generated by $s_{\alpha}$ in $W$. Let $R \llbracket M \rrbracket_{F}^{W_{\alpha}}$ denote the subring of fixed elements of $R \llbracket M \rrbracket_{F}$ under the action of $W_{\alpha}$.

If the ring $R$ has no 2-torsion, then the operators $\Delta_{\alpha}$ and $C_{\alpha}$ are $R \llbracket M \rrbracket_{F}^{W}$-linear. In particular, they are $R \llbracket M \rrbracket_{F}^{W}$-linear.

Proof. - For the operator $\Delta_{\alpha}$, by Formula (4) of 3.8 it suffices to show that $\Delta_{\alpha}(u)=0$ for $u \in R \llbracket M \rrbracket_{F}^{W_{\alpha}}$. If $R$ and thus $R \llbracket M \rrbracket_{F}$ has no 2-torsion, it is equivalent to $\Delta_{\alpha}(u)=-\Delta_{\alpha}(u)$, which holds by Formula (3) of 3.8.

The same facts for $C_{\alpha}$ then follow from its definition in terms of $\Delta_{\alpha}$.

Lemma 3.15. - Assume $R$ has no 2-torsion. Then for any root $\alpha$, the element $x_{\alpha}$ is regular in $R \llbracket M \rrbracket_{F}$ and the set of elements $u$ such that $\Delta_{\alpha}(u)=0$ is $R \llbracket M \rrbracket_{F}^{W_{\alpha}}$.

Proof. - Let $\omega_{1}, \ldots, \omega_{n}$ be the fundamental weights corresponding to a choice of simple roots $\alpha=\alpha_{1}, \ldots, \alpha_{n}$ of the root system. Possibly extending $M$, we can assume that the $\omega_{i}$ are a basis of $M$. By the isomorphism $R \llbracket M \rrbracket_{F} \simeq R \llbracket x_{\omega_{1}}, \ldots, x_{\omega_{n}} \rrbracket$ of Corollary 2.13, $x_{\alpha}$ becomes $\sum_{i}<\alpha, \alpha_{i}>x_{\omega_{i}}+u, u \in \mathcal{J}^{2}$. One easily checks that this element is regular if at least one of the $\left\langle\alpha, \alpha_{i}\right\rangle$ is regular in $R$. In particular, this is the case if $\left\langle\alpha_{1}, \alpha_{1}\right\rangle=2$ is regular in $R$. By Equation (1) of Proposition 3.8, the last part of the claim is then clear.

Proposition 3.16. - Let $f: R \rightarrow R^{\prime}$ be a ring morphism sending a formal group law $F$ over $R$ to a formal group law $F^{\prime}$ over $R^{\prime}$. Then, the operators $\Delta$ and $C$ satisfy

$$
f_{*} \Delta_{\alpha}^{F}=\Delta_{\alpha}^{F^{\prime}} f_{*} \quad \text { and } \quad f_{*} C_{\alpha}^{F}=C_{\alpha}^{F^{\prime}} f_{*}
$$

where $f_{*}: R \llbracket M \rrbracket_{F} \rightarrow R^{\prime} \llbracket M \rrbracket_{F^{\prime}}$ is the morphism of Lemma 2.6 .

Proof. - This is clear by construction.

\section{Endomorphisms of a formal group ring}

In the present section we introduce and study the subalgebra $\mathscr{D}(M)_{F}$ of $R$-linear endomorphisms of a formal group ring $R \llbracket M \rrbracket_{F}$ generated by the $\Delta$ operators of the previous section. The main result (Theorem 4.11) says that operators $\Delta_{I_{w}}^{F}$, where $w$ runs through all elements of the Weyl group and $I_{w}$ is any chosen reduced decomposition of $w$, form a basis of $\mathscr{D}(M)_{F}$ as an $R \llbracket M \rrbracket_{F}$-module.

4.1. - Let $R \llbracket M \rrbracket_{F}$ be a formal group ring corresponding to a formal group law $F$ over a ring $R$ and an Abelian group $M$ (see Def. 2.4). Let $J_{F}$ be the kernel of the augmentation map $R \llbracket M \rrbracket_{F} \rightarrow R$ as in 2.5. By convention, we set $\mathcal{J}_{F}^{i}=R \llbracket M \rrbracket_{F}$ for any $i \leq 0$. We define the associated graded ring

$$
\mathscr{G} r_{R}^{*}(M)_{F}=\bigoplus_{i=0}^{\infty} \mathcal{J}_{F}^{i} / \mathcal{J}_{F}^{i+1}
$$

Lemma 4.2. - The morphism of graded R-algebras

$$
\phi: S_{R}^{*}(M) \rightarrow \mathscr{G} r_{R}^{*}(M)_{F}
$$

defined by sending $\lambda$ to $x_{\lambda}$ is an isomorphism.

4 e SÉRIE - TOME $46-2013$ - No 3 
Proof. - The map is well-defined since $x_{\lambda+\mu}-\left(x_{\lambda}+x_{\mu}\right)$ is in $\mathcal{J}_{F}^{2}$, and it is obviously surjective. Let us define a map in the other direction. Recall that by definition $R \llbracket M \rrbracket_{F}=$ $R \llbracket x_{M} \rrbracket / \mathscr{J}_{F}$. Let $\tilde{\mathscr{J}}$ denote the kernel of the augmentation map $R \llbracket x_{M} \rrbracket \rightarrow R$ (see 2.5). Then we have

$$
J_{F}=\tilde{J} / \mathscr{J}_{F} \text { and } \mathcal{J}_{F}^{i} / \mathcal{J}_{F}^{i+1}=\tilde{I}^{i} /\left(\mathcal{J}_{F} \cap \tilde{I}^{i}+\tilde{I}^{i+1}\right) \text {. }
$$

Since $\tilde{I}^{i} / \tilde{I}^{i+1}$ is given by monomials of degree $i$, we may define a map of $R$-modules

$$
\psi_{i}: \tilde{I}^{i} / \tilde{I}^{i+1} \rightarrow S_{R}^{i}(M)
$$

by sending a monomial of degree $i$ in some $x_{\lambda}$-s to the symmetric product of the $\lambda$-s involved. This map passes to the quotient since $\left(\mathscr{J}_{F} \cap \tilde{I}^{i}+\tilde{I}^{i+1}\right) / \tilde{J}^{i+1}$ is generated as an $R$-module by elements of the form $\rho$. $\left(x_{\lambda}+x_{\mu}-x_{\lambda+\mu}\right)$ where $\rho$ is a monomial of degree $i-1$. The sum $\oplus_{i} \psi_{i}$ passes through the quotient as well and, hence, gives the desired inverse map.

4.3. - Consider now a reduced root datum on $M$ as in Section 3 and let $R \llbracket M \rrbracket_{F}$ be the associated formal group ring. Consider the operators $\Delta_{\alpha}^{F}$ introduced in Def. 3.5. By their very definition, the operators $\Delta_{\alpha}^{F}$ send $\mathcal{J}_{F}^{i}$ to $\mathcal{J}_{F}^{i-1}$. Hence, they induce $R$-linear operators of degree -1 on the graded ring $\mathscr{G} r_{R}^{*}(M)_{F}$, denoted by $\mathscr{G} \Delta_{\alpha}^{F}$. Similarly, we define graded versions of operators $C_{\alpha}^{F}$ (see Def. 3.12) on $\mathscr{G} r_{R}^{*}(M)_{F}$, denoted by $\mathscr{G} C_{\alpha}^{F}$.

Proposition 4.4. - The isomorphism $\phi$ of Lemma 4.2 exchanges the operator $\operatorname{Gr} \Delta_{\alpha}^{F}$ (resp. $\operatorname{Gr} C_{\alpha}^{F}$ ) on $\mathscr{G} r_{R}^{*}(M)_{F}$ with $\Delta_{\alpha}^{F_{a}}\left(\right.$ resp. $\left.-\Delta_{\alpha}^{F_{a}}\right)$ on the symmetric algebra $S_{R}^{*}(M)$, where $F_{a}$ denotes the additive formal group law as in Example 2.19 and the $\Delta_{\alpha}^{F_{a}}=-C_{\alpha}^{F_{a}}$ are the classical operators of [7].

Proof. - Induction on the degree using (4) of Prop. 3.8.

Definition 4.5. - Let $\mathscr{D}(M)_{F}$ be the subalgebra of $R$-linear endomorphisms of $R \llbracket M \rrbracket_{F}$ generated by the $\Delta_{\alpha}^{F}$ for all roots $\alpha$ and by multiplications by elements of $R \llbracket M \rrbracket_{F}$. Note that by Formula (1) of Proposition 3.8, $\mathscr{D}(M)_{F}$ contains $s_{\alpha}$ and it contains $C_{\alpha}^{F}$ by its definition. Let $\mathscr{D}(M)_{F}^{(i)}$ be the sub $R \llbracket M \rrbracket_{F}$-module of $\mathscr{D}(M)_{F}$ generated by $u \Delta_{\alpha_{1}}^{F} \cdots \Delta_{\alpha_{n}}^{F}$, where $u \in \mathcal{I}_{F}^{m}$ and $m-n \geq i$. This defines a filtration on $\mathscr{D}(M)_{F}$

$$
\mathscr{D}(M)_{F} \supseteq \cdots \supseteq \mathscr{D}(M)_{F}^{(i)} \supseteq \mathscr{D}(M)_{F}^{(i+1)} \supseteq \cdots \supseteq 0
$$

with the property that $\mathscr{D}(M)_{F}=\cup_{i} \mathscr{D}(M)_{F}^{(i)}$. We define the associated graded $R \llbracket M \rrbracket_{F^{-}}$ module

$$
\mathscr{G} r^{*} \mathscr{D}(M)_{F}=\bigoplus_{i=-\infty}^{\infty} \mathscr{D}(M)_{F}^{(i)} / \mathscr{D}(M)_{F}^{(i+1)}
$$

Proposition 4.6. - The filtration on $\mathscr{D}(M)_{F}$ above has the following properties:

1. For any root $\alpha$, we have

$$
\Delta_{\alpha}^{F} \mathscr{D}(M)_{F}^{(i)} \subseteq \mathscr{D}(M)_{F}^{(i-1)}, \quad \mathscr{D}(M)_{F}^{(i)} \Delta_{\alpha}^{F} \subseteq \mathscr{D}(M)_{F}^{(i-1)}
$$

and similarly for $C_{\alpha}^{F}$.

2. For any integer $j$, we have $\mathcal{J}_{F}^{j} \mathscr{D}(M)_{F}^{(i)} \subseteq \mathscr{D}(M)_{F}^{(i+j)}$.

3. For any operator $D \in \mathscr{D}(M)_{F}^{(i)}$, we have $D\left(\mathcal{J}_{F}^{j}\right) \subseteq \mathcal{J}_{F}^{i+j}$. 
4. $\cap_{i} \mathscr{D}(M)_{F}^{(i)}=\{0\}$.

Proof. - The claim (1) follows from Equation (4) in Prop. 3.8 (resp. Equation (4) in Prop. 3.13). The claim (2) is obvious. The claim (3) follows from the fact that $\Delta_{\alpha}^{F}\left(\mathcal{J}_{F}^{i}\right) \subseteq \mathcal{J}_{F}^{i-1}$. To prove (4) observe that any $D$ in $\cap_{i} \mathscr{D}(M)_{F}^{(i)}$ sends $R \llbracket M \rrbracket_{F}$ to $\cap_{i} \mathcal{J}_{F}^{i}=0$ by (3) so is the zero operator.

4.7. - By claim (3) of Prop. 4.6 the graded module $\mathscr{G} r^{*} \mathscr{D}(M)_{F}$ acts by graded endomorphisms on the graded ring $\mathscr{G} r_{R}^{*}(M)_{F}$, which is isomorphic to $S_{R}^{*}(M)$ by Lemma 4.2. Let $F_{a}$ denote the additive formal group law. Then the associated subalgebra $\mathscr{D}=\mathscr{D}(M)_{F_{a}}$ coincides with one considered in [7, §3]. By Prop. 4.4, the class of $\Delta_{\alpha}^{F}$ (resp. $C_{\alpha}^{F}$ ) in $\mathscr{G r}^{(-1)} \mathscr{D}(M)_{F}$ acts by $\Delta_{\alpha}^{F_{a}}$ (resp. $-\Delta_{\alpha}^{F_{a}}$ ) on $S_{R}^{*}(M)$ and the class of the multiplication by $x_{\alpha}$ in $\mathscr{G} r^{(1)} \mathscr{D}(M)_{F}$ acts by the multiplication by $\alpha$ on $S_{R}^{*}(M)$.

Proposition 4.8. - The graded module $\mathscr{G} r^{*} \mathscr{D}(M)_{F}$ over the graded ring $\mathscr{G} r_{R}^{*}(M)_{F} \simeq$ $S_{R}^{*}(M)$ is isomorphic to $\mathscr{D}$.

Proof. - It is a sub-module of the $R$-linear endomorphisms of $\mathscr{G} r^{*} R \llbracket M \rrbracket_{F}$ generated by the same elements as $\mathscr{D}$.

4.9. - Let $A$ be a ring and let $I$ be an ideal of $A$, such that $A$ is complete and Hausdorff for the $\mathscr{J}$-adic topology. Let $M$ be an $A$-module together with a $\mathscr{J}$-filtration $\left(M^{(i)}\right)_{i \in \mathbb{Z}}$, i.e., $\mathscr{I} M^{(i)} \subseteq M^{(i+1)}$ for any $i$. Consider the associated graded $\operatorname{ring} \mathscr{G} r^{*} A$ and the associated graded $\mathscr{G} r^{*} A$-module $\mathscr{G} r^{*} M$.

The filtration is called exhaustive (see [2, III, §2, 1]) if any $\lambda \in M$ belongs to some $M^{(i)}$, i.e., $M=\cup_{i} M^{(i)}$. For an exhaustive filtration let $\nu(\lambda)$ denote the largest integer such that $\lambda \in M^{\nu(\lambda)}$ and let $\bar{\lambda}$ denote the class of $\lambda$ in $M^{(\nu(\lambda))} / M^{(\nu(\lambda)+1)}$ if $\nu(\lambda)$ exists and 0 if $\nu(\lambda)$ does not exist. Note that $\bar{\lambda}$ is nonzero if and only if $\lambda$ is not in $\cap_{i} M^{(i)}$.

Lemma 4.10. - Let $M$ be an A-module with an exhaustive I-filtration. Let $\lambda_{1}, \ldots, \lambda_{n}$ be elements of $M$. Then

1. Assume $A$ is complete for the I-adic topology and $M$ is Hausdorff. If $\bar{\lambda}_{1}, \ldots, \bar{\lambda}_{n}$ generate $\mathscr{G} r^{*} M$ as a $\mathscr{G} r^{*} A$-module, then $\lambda_{1}, \ldots, \lambda_{n}$ generate $M$ as an A-module.

2. Assume $A$ is Hausdorff. If $\bar{\lambda}_{1}, \ldots, \bar{\lambda}_{n}$ are independent elements in the $\mathscr{G} r^{*} A$-module $\mathscr{G} r^{*} M$, then $\lambda_{1}, \ldots, \lambda_{n}$ are independent in the A-module $M$.

3. Assume $A$ is Hausdorff complete and $M$ is Hausdorff. If $\mathscr{G}{ }^{*} M$ is a finitely generated free $\mathcal{G} r^{*} A$-module, then $M$ is a finitely generated free A-module.

Proof. - Let $A[i]$ be $A$ itself considered as an $A$-module with the shifted filtration $g^{j+\nu\left(\lambda_{i}\right)}$ (by convention, $g^{j}=A$ for $j \leq 0$ ). The claims (1) and (2) then follow from [3, III, §2, 8, Cor. 1 and 2] applied to $X=\bigoplus_{i} A[i]$, to $Y=M$ and to $\bigoplus_{i} A[i] \rightarrow M$ equal to the map sending $\left(a_{i}\right)_{i}$ to $\sum_{i} a_{i} \lambda_{i}$. The last claim (3) is an immediate consequence of (1) and (2).

We now come to the main result of this section:

$4^{\text {e }}$ SÉRIE - TOME $46-2013-\mathrm{N}^{\mathrm{o}} 3$ 
THEOREM 4.11. - Let $\mathscr{D}(M)_{F}$ be the algebra of operators defined above. For each element $w \in W$, choose a reduced decomposition $I_{w}$ in simple reflections. Then the operators $\Delta_{I_{w}}^{F}$ (resp. $C_{I_{w}}^{F}$ ) form a basis of $\mathscr{D}(M)_{F}$ as an $R \llbracket M \rrbracket_{F}$-module.

Proof. - When the formal group law is additive, this is proved in [7, $\S 4$, Cor. 1] for the $\Delta_{w}^{F_{a}}$ (and $C_{w}^{F_{a}}=(-1)^{l(w)} \Delta_{w}^{F_{a}}$ ). The filtered module $\mathscr{D}(M)_{F}$ is Hausdorff by part (4) of Proposition 4.6, and $R \llbracket M \rrbracket_{F}$ is complete. We can therefore apply part (3) of Lemma 4.10 to deduce the general case from the corresponding fact on the associated graded objects, which is the additive case by Proposition 4.8.

\section{Torsion indices and augmented operators}

In the present section we study the augmentation $\epsilon \mathscr{D}(M)_{F}$ of the algebra of operators $\mathscr{D}(M)_{F}$. The main result is Proposition 5.4 which is what becomes of Theorem 4.11 when the augmentation $\epsilon$ is applied. Similarly, we then examine the filtration on $\epsilon \mathscr{D}(M)_{F}$ obtained by applying $\epsilon$ to the one on $\mathscr{D}(M)_{F}$ introduced in 4.5 .

5.1. - Consider the torsion index $\mathfrak{t}$ of the root datum as defined in $[7, \S 5]$ and its prime divisors, called torsion primes. It has been computed for simply connected root data of all types (see [7, §7, Prop. 8] and [20, 21]). The results of these computations are summarized in the following table:

\begin{tabular}{l||c|c|c|c|c|c|c|c|c} 
Type & $A_{l}$ & $B_{l}, l \geq 3$ & $C_{l}$ & $D_{l}, l \geq 4$ & $G_{2}$ & $F_{4}$ & $E_{6}$ & $E_{7}$ & $E_{8}$ \\
\hline Torsion primes & $\varnothing$ & 2 & $\varnothing$ & 2 & 2 & 2,3 & 2,3 & 2,3 & $2,3,5$ \\
Torsion index & 1 & $2^{e(l)}$ & 1 & $2^{e(l-1)}$ & 2 & $2 \cdot 3$ & $2 \cdot 3$ & $2^{2} \cdot 3$ & $2^{6} \cdot 3^{2} \cdot 5$
\end{tabular}

The exponent $e(l)$ is equal to $l-\left\lfloor\log _{2}\left(\left(\begin{array}{c}l+1 \\ 2\end{array}\right)+1\right)\right\rfloor$ except for certain values of $l$ equal to or slightly larger than a power of 2 which are explicitly given in [21, Thm. 0.1]. Since $\mathfrak{t}\left(\mathscr{R}_{1} \times \mathscr{R}_{2}\right)=\mathfrak{t}\left(\mathscr{R}_{1}\right) \cdot \mathfrak{t}\left(\mathscr{R}_{2}\right)$, we may compute torsion indices of semi-simple root data by reducing to irreducible ones.

5.2. - Let $M$ be the character group of the root datum of $G$ and let $F$ be a formal group law over a ring $R$. Let $\epsilon: R \llbracket M \rrbracket_{F} \rightarrow R$ be the augmentation map with the kernel $\nearrow_{F}$. Let $N$ be the length of the longest element $w_{0}$ of the Weyl group $W$ of $G$. Let $F_{a}$ be an additive formal group law.

By definition of the torsion index (see [7, §5]) there exists an element $a$ of the symmetric algebra $S_{R}^{N}(M)$ such that

$$
\Delta_{w_{0}}^{F_{a}}(a)=\mathfrak{t}
$$

Let $\phi: S_{R}^{*}(M) \stackrel{\simeq}{\rightarrow} \mathscr{G} r_{R}^{*}(M)_{F}$ be an isomorphism from Lemma 4.2. Let $u_{0} \in \mathcal{I}_{F}^{N}$ be an element such that $\bar{u}_{0}=\phi(a)$. Therefore, for any reduced decomposition $I_{0}$ of $w_{0}$ we have $\epsilon \Delta_{I_{0}}^{F}\left(u_{0}\right)=\mathfrak{t}$ or, in other words, by Prop. 4.4

$$
\Delta_{I_{0}}^{F}\left(u_{0}\right)=\mathfrak{t}+g_{F} \quad \text { and } \quad C_{I_{0}}^{F}\left(u_{0}\right)=(-1)^{N} \mathfrak{t}+g_{F} \quad \text { in } R \llbracket M \rrbracket_{F} .
$$

Lemma 5.3. - Let I be a sequence of simple reflections and let $l(I)$ be its length. Then

1. for any $i \in \mathbb{Z}, \Delta_{I}^{F}\left(\mathcal{J}_{F}^{i}\right) \subseteq \mathcal{J}_{F}^{i-l(I)}$ and $C_{I}^{F}\left(\mathcal{J}_{F}^{i}\right) \subseteq \mathcal{J}_{F}^{i-l(I)}$; 
2. if $l(I) \leq i$ and $I$ is not reduced, then

$$
\Delta_{I}^{F}\left(\mathcal{J}_{F}^{i}\right) \subseteq \mathcal{J}_{F}^{i-l(I)+1} \quad \text { and } \quad C_{I}^{F}\left(\mathcal{J}_{F}^{i}\right) \subseteq \mathcal{J}_{F}^{i-l(I)+1} ;
$$

3. let $u_{0} \in \mathcal{J}_{F}^{N}$ be the element chosen above. If $l(I) \leq N$ then

$$
\epsilon \Delta_{I}^{F}\left(u_{0}\right)=(-1)^{N} \epsilon C_{I}^{F}\left(u_{0}\right)= \begin{cases}\mathfrak{t} & \text { if } I \text { is reduced and } l(I)=N \\ 0 & \text { otherwise. }\end{cases}
$$

Proof. - Claim (1) follows from Prop. 4.6, (1). To check (2), it therefore suffices to check that $\operatorname{Gr} \Delta_{I}^{F}$ is zero. It follows from the corresponding fact in the additive case [7, §4, Prop. 3, (a)] after applying Prop. 4.4. The "otherwise" case of (3) follows from (2). The case where $I$ is reduced and has length $N$ follows from the definition of $u_{0}$.

Proposition 5.4. - Consider the R-module $\epsilon \mathscr{D}(M)_{F}$ of all R-linear forms $\epsilon D$, where $D \in \mathscr{D}(M)_{F}$. Assume the torsion index $\mathfrak{t}$ is regular in $R$. Then for any choice of a collection of reduced decomposition $I_{w}$ for each $w \in W$, the $\left(\epsilon \Delta_{I_{w}}\right)_{w \in W}$ (resp. the $\left(\epsilon C_{I_{w}}\right)_{w \in W}$ ) form an $R$-basis of $\epsilon \mathscr{D}(M)_{F}$.

Proof. - By Theorem 4.11, the $\epsilon \Delta_{I_{w}}$ generate $\epsilon \mathscr{D}(M)_{F}$. We prove that the $\epsilon \Delta_{I_{w}}$ such that $l\left(I_{w}\right) \leq i$ are independent by induction on $i$. It is obviously true when $i<0$. Let $\sum_{l(w) \leq i} r_{w} \epsilon \Delta_{I_{w}}=0$. By induction, it suffices to show that the coefficients $r_{w}$ with $l(w)=i$ are zero. For any $v$ and $w$ of length $i$, the concatenation $I_{w}+I_{v^{-1} w_{0}}$ is a reduced decomposition of $w_{0}$ if and only if $v=w$. Thus, by Lemma 5.3 (1) and (3) we have $\sum_{l(w) \leq i} r_{w} \in \Delta_{I_{w}}\left(\Delta_{I_{v-1} w_{0}}\left(u_{0}\right)\right)=r_{v} \mathfrak{t}$ which implies that $r_{v}=0$ by regularity of $\mathfrak{t}$. The same proof works for the $C_{I_{w}}$.

Lemma 5.5. - Assume $\mathfrak{t}$ is regular in $R$.

1. For any reduced decomposition $I_{w}$ of $w$ and any element $u \in \mathcal{J}_{F}^{i} \backslash \mathcal{J}_{F}^{i+1}$, the element $u \Delta_{I_{w}}^{F}$ is in $\mathscr{D}(M)_{F}^{(i-l(w))} \backslash \mathscr{D}(M)_{F}^{(i-l(w)+1)}$.

2. The group $\mathscr{D}(M)_{F}^{(i)}$ is the set of $D \in \mathscr{D}(M)_{F}$ such that $D\left(\mathcal{J}_{F}^{j}\right) \subseteq \mathcal{I}_{F}^{i+j}$.

Proof. - It suffices to prove (1) after inverting the torsion index. By Lemma 5.3 (1), the operator $u \Delta_{I_{w}}^{F}$ is in $\mathscr{D}(M)_{F}^{(i-l(w))}$. Consider the element $y=\Delta_{I_{w^{-1}} w_{0}}^{F}\left(u_{0}\right)$. It is in $\mathcal{I}_{F}^{l(w)}$. By Lemma 5.3 (3) the element $s=\Delta_{I_{w}}^{F}(y)$ is invertible. Hence, $u \Delta_{I_{w}}^{F}(y)=u s$ cannot be in $\mathcal{J}_{F}^{i+1}$, and therefore $u \Delta_{I_{w}}^{F}$ is not in $\mathscr{D}(M)_{F}^{(i-l(w)+1)}$.

To prove (2), first note that Prop. 4.6 (3) immediately gives one inclusion. Suppose $D$ is such that $D\left(\mathcal{J}_{F}^{j}\right) \subseteq \mathcal{J}_{F}^{i+j}$. Using Theorem 4.11 we may write it as

$$
D=\sum_{w} u_{w} \Delta_{I_{w}}^{F}
$$

Recall that $\nu\left(u_{w}\right)$ denotes the largest integer such that $u_{w} \in \mathcal{I}_{F}^{v}$ (see 4.9). Consider the set $S$ of $w \in W$ such that $\nu\left(u_{w}\right)-l(w)$ is minimal and take an element $w_{1} \in S$ such that $l\left(w_{1}\right)$ is maximal. To show that $D \in \mathscr{D}(M)_{F}^{(i)}$, it suffices to show that $\nu\left(u_{w_{1}}\right)-l\left(w_{1}\right) \geq i$ by minimality of elements of $S$. 
Let $y_{1}=\Delta_{I_{w_{1}^{-1} w_{0}}^{F}}^{F}\left(u_{0}\right)$ be the corresponding element in $g^{l\left(w_{1}\right)}$. We have

$$
D\left(y_{1}\right)=u_{w_{1}} \Delta_{I_{w_{1}}}^{F}\left(y_{1}\right)+\sum_{w \in S \backslash\left\{w_{1}\right\}} u_{w} \Delta_{I_{w}}\left(y_{1}\right)+\sum_{w \notin S} u_{w} \Delta_{I_{w}}\left(y_{1}\right) .
$$

By Lemma 5.3 (1) and (2), two sums on the right are in $\mathcal{J}_{F}^{\nu\left(u_{w_{1}}\right)+1}$. As in the proof of (1), $u_{w_{1}} \Delta_{I_{w_{1}}}^{F}\left(y_{1}\right)$ is not in $g^{\nu\left(u_{w_{1}}\right)+1}$ so $D\left(y_{1}\right)$ is in $\mathcal{J}_{F}^{\nu\left(u_{w_{1}}\right)} \backslash \mathcal{J}_{F}^{\nu\left(u_{w_{1}}\right)+1}$. Since $D\left(y_{1}\right)$ is also in $\mathcal{J}_{F}^{l\left(w_{1}\right)+i}$, we obtain that $\nu\left(u_{w_{1}}\right)-l\left(w_{1}\right) \geq i$.

We now consider the filtration on $\epsilon \mathscr{D}(M)_{F}$ that is the image by $\epsilon$ of the filtration on $\mathscr{D}(M)_{F}$ defined in 4.5, i.e.,

$$
\epsilon \mathscr{D}(M)_{F} \supseteq \cdots \supseteq \epsilon \mathscr{D}(M)_{F}^{(i)} \supseteq \epsilon \mathscr{D}(M)_{F}^{(i+1)} \supseteq \cdots \supseteq 0 .
$$

Proposition 5.6. - Assume $\mathfrak{t}$ is regular in $R$. This filtration satisfies the following.

1. For any $i \in \mathbb{Z}$ and $\epsilon D \in \epsilon \mathscr{D}(M)_{F}^{(i)}$, we have $\epsilon D\left(\mathcal{J}_{F}^{-i+1}\right)=0$.

2. Let $E$ be a subset of $W$ of elements of length $l$, and let the $I_{w}$ be reduced decompositions of each of the $w \in E$. Then any nonzero $R$-linear combination

$$
f=\sum_{w \in E} r_{w} \cdot \epsilon \Delta_{I_{w}}^{F} \quad\left(\text { resp. } \epsilon C_{I_{w}}^{F}\right)
$$

is in $\epsilon \mathscr{D}(M)_{F}^{-l} \backslash \epsilon \mathscr{D}(M)_{F}^{-l+1}$. In particular, for any $w$

$$
\epsilon \Delta_{I_{w}}^{F} \in \epsilon \mathscr{D}(M)_{F}^{-l(w)} \backslash \epsilon \mathscr{D}(M)_{F}^{-l(w)+1} \quad \text { (resp. with } C_{I_{w}}^{F} \text { ). }
$$

3. For any choice of reduced decompositions $I_{w}$ for every element $w \in W$, the $R$-module $\epsilon \mathscr{D}(M)_{F}^{(-i)}$ is a free $R$-module with basis the $\epsilon \Delta_{I_{w}}$ (resp. $\epsilon C_{I_{w}}$ ) with $l(w) \leq i$.

4. For any $i \leq-N$, we have $\epsilon \mathscr{D}(M)_{F}^{(i)}=\epsilon \mathscr{D}(M)_{F}$ and for any $i>0$, we have $\epsilon \mathscr{D}(M)_{F}^{(i)}=0$, i.e., the filtration is of the form

$$
\epsilon \mathscr{D}(M)_{F}=\epsilon \mathscr{D}(M)_{F}^{(-N)} \supseteq \epsilon \mathscr{D}(M)_{F}^{(-N+1)} \supseteq \cdots \supseteq \epsilon \mathscr{D}(M)_{F}^{(0)} \supseteq 0 .
$$

Proof. - Claim (1) follows from Lemma 5.5 (2). To prove (2), we first apply $f$ to $\Delta_{I_{w^{-1} w_{0}}}^{F}\left(u_{0}\right)$. By Lemma 5.3 (3), we obtain $\mathfrak{t} r_{w}$, which has to be zero by (1) for the element $f$ to be in $\mathcal{J}_{F}^{l+1}$. Therefore, each $r_{w}$ is zero. The last two claims follow.

\section{Invariants and the characteristic map}

We now come to the definition of an algebraic replacement $\mathcal{H}(M)_{F}$ for the oriented cohomology $\mathrm{h}(G / B)$ when $G$ is a split semi-simple simply connected algebraic group corresponding to the root datum and $B$ is a Borel of $G$. The identification of $\mathcal{H}(M)_{F}$ with $\mathrm{h}(G / B)$ is the subject of Section 13.

Definition 6.1. - Let $\mathcal{H}(M)_{F}$ be the $R$-dual of $\epsilon \mathscr{D}(M)_{F}$ and let

$$
\mathfrak{c}^{F}: R \llbracket M \rrbracket_{F} \rightarrow \mathcal{H}(M)_{F}
$$

denote the natural map obtained by duality, i.e., sending $u$ to the evaluation at $u$. When the formal group law is clear from the context, we write $\mathfrak{c}$ for $\mathfrak{c}^{F}$. 
Again, when $F=F_{a}$ is additive, this $\mathcal{H}(M)_{F}$ corresponds to the one defined by Demazure in $[7, \S 3]$.

REMARK 6.2. - Observe that when $R$ has no 2-torsion, since the operators $\Delta_{\alpha}^{F}$ are $R \llbracket M \rrbracket_{F}^{W}$-linear by Proposition 3.14, the characteristic map satisfies $\mathfrak{c}(f u)=\epsilon(f) \mathfrak{c}(u)$ when $f$ is in $R \llbracket M \rrbracket_{F}^{W}$.

THEOREM 6.3. - Let $\left(I_{w}\right)_{w \in W}$ be a choice of reduced decompositions and let $\Delta_{I_{w}}^{F}$ be the operator defined in 3.9. When the torsion index $\mathfrak{t}$ is regular in $R$, there is a unique $R$-basis $z_{I_{w}}^{\Delta}$ of $\mathcal{H}(M)_{F}$ such that the characteristic map is given by

$$
\mathfrak{c}(u)=\sum_{w} \epsilon \Delta_{I_{w}}^{F}(u) z_{I_{w}}^{\Delta} .
$$

Similarly, there is a unique $R$-basis $z_{I_{w}}^{C}$ of $\mathcal{H}(M)_{F}$ such that the characteristic map is given by

$$
\mathfrak{c}(u)=\sum_{w} \epsilon C_{I_{w}}^{F}(u) z_{I_{w}}^{C} .
$$

Proof. - We take the bases that are dual to the ones of Proposition 5.4.

Theorem 6.4. - Assume that the torsion index $\mathfrak{t}$ is invertible in $R$. Then for any choice of a collection of reduced decompositions $I_{w}$ for every $w \in W$, the $\mathfrak{c}\left(\Delta_{I_{w}}\left(u_{0}\right)\right)$ (resp. the $\left.\mathfrak{c}\left(C_{I_{w}}\left(u_{0}\right)\right)\right)$ form an $R$-basis of the image of $\mathfrak{c}$. In particular, the characteristic map $\mathfrak{c}$ is surjective.

Proof. - We have $\mathfrak{c}\left(u_{0}\right)=\mathfrak{t} . z_{I_{0}}^{\Delta}$ and

$$
\mathfrak{c}\left(\Delta_{I_{w^{-1} w_{0}}}\left(u_{0}\right)\right)=\mathfrak{t} \cdot z_{I_{w}}^{\Delta}+\sum_{l(v)>l(w)} \epsilon \Delta_{I_{v}} \Delta_{I_{w^{-1} w_{0}}}\left(u_{0}\right) z_{I_{v}}^{\Delta}
$$

by part (3) of Lemma 5.3. All the $z_{I_{w}}^{\Delta}$ are thus in the image of the characteristic map by decreasing induction on the length of $w$. The same proof works when replacing $\Delta$ by $C$.

Still assuming that $\mathfrak{t}$ is regular in $R$, let $f: R \rightarrow R^{\prime}$ be a ring morphism sending a formal group law $F$ over $R$ to a formal group law $F^{\prime}$ over $R^{\prime}$. By restriction (through $f$ ), $\mathcal{H}_{R^{\prime}}(M)_{F^{\prime}}$ is a $R$-module. Let $f_{\mathscr{H}}: \mathscr{H}(M)_{F} \rightarrow \mathcal{H}_{R^{\prime}}(M)_{F^{\prime}}$ be the $R$-linear map sending the element $z_{I_{w}}^{\Delta}$ in $\mathcal{H}(M)_{F}$ to the corresponding one in $\mathcal{H}_{R^{\prime}}(M)_{F^{\prime}}$.

Proposition 6.5. - We have the following:

1. The assignment $f \rightarrow f_{\mathcal{H}}$ defines a functor from the category of formal group laws $(R, F)$ ( $\mathfrak{t}$ regular in $R$ ) to the category of pairs $(R, M)$ where $M$ is a module over $R$, and the morphisms are the obvious ones.

2. We have $f_{\mathscr{H}} \mathfrak{c}^{F}=\mathfrak{c}^{F^{\prime}} f_{*}$.

3. The map $f_{\mathscr{H}}$ defined above is independent of the choice of the reduced decompositions $I_{w}$.

Proof. - Point (1) holds by construction. Point (2) follows from the formula for the characteristic map given in Theorem 6.3. Since $f_{*}$ is defined independently of the $I_{w}$, the last item (3) holds if the characteristic map is surjective. The general case follows by embedding $\mathscr{H}_{R}(M)_{F}$ into $\mathscr{H}_{R[1 / \mathrm{t}]}(M)_{F}$ where the characteristic map is surjective by Theorem 6.4.

$4^{\mathrm{e}}$ SÉRIE - TOME $46-2013-\mathrm{N}^{\circ} 3$ 
Proposition 6.6. - Assume that $\mathfrak{t}$ is invertible in $R$. Fix a reduced decomposition $I_{w}$ for each $w \in W$. Then

1. For any $x \in R \llbracket M \rrbracket_{F}$, the system of linear equations in $R \llbracket M \rrbracket_{F}$

$$
\Delta_{I_{v}}(x)=\sum_{w \in W} r_{w} \Delta_{I_{v}} \Delta_{I_{w}}\left(u_{0}\right)
$$

for all $v$ in $W$, has a unique solution $\left(r_{w}\right)_{w \in W}$.

2. If $\left(r_{w}\right)_{w \in W}$ is the solution of (1), then for any $D \in \mathscr{D}(M)_{F}$, we have the equality

$$
D(x)=\sum_{w \in W} r_{w} D \Delta_{I_{w}}\left(u_{0}\right) .
$$

3. If $\left(r_{w}\right)_{w \in W}$ is the solution of (1) and if $R$ has no 2-torsion, then all the $r_{w}$ are in fact in $R \llbracket M \rrbracket_{F}^{W}$.

The same is true when replacing $\Delta$ by $C$ everywhere in (1), (2) and (3).

Proof. - Let $A$ be a ring and $\nearrow$ be an ideal of $A$ contained in its Jacobson radical. A matrix with coefficients in $A$ is invertible if and only if the corresponding matrix with coefficients in $A / \mathcal{J}$ is invertible. Since $\nearrow_{F}$ is contained in the radical of $R \llbracket M \rrbracket_{F}$, it suffices to show that the matrix of the system in $R \llbracket M \rrbracket_{F} / \mathscr{J}_{F}$ is invertible. If we order the $v$ 's by increasing length and the $w$ 's by decreasing length, Lemma 5.3 shows that the matrix is lower triangular with $\mathfrak{t}$ on the diagonal, and it is therefore invertible. Part (2) follows from part (1) since the $\Delta_{I_{w}}$ (resp. the $C_{I_{w}}$ ) form a basis of $\mathscr{D}(M)_{F}$ as an $R \llbracket M \rrbracket_{F}$-module by Theorem 4.11. Let us prove part (3). For any simple root $\alpha$ and for any $v \in W$, we have

$$
\begin{aligned}
\Delta_{\alpha} \Delta_{I_{v}}(x) & =\sum_{w \in W} \Delta_{\alpha}\left(r_{w} \Delta_{I_{v}} \Delta_{I_{w}}\left(u_{0}\right)\right) \\
& =\sum_{w \in W} r_{w} \Delta_{\alpha} \Delta_{I_{v}} \Delta_{I_{w}}\left(u_{0}\right)+\sum_{w \in W} \Delta_{\alpha}\left(r_{w}\right) s_{\alpha} \Delta_{I_{v}} \Delta_{I_{w}}\left(u_{0}\right)
\end{aligned}
$$

using part (4) of Proposition 3.8. But we also have

$$
\Delta_{\alpha} \Delta_{I_{v}}(x)=\sum_{w \in W} r_{w} \Delta_{\alpha} \Delta_{I_{v}} \Delta_{I_{w}}\left(u_{0}\right)
$$

by (2) with $D=\Delta_{\alpha} \Delta_{I_{v}}$. So

$$
\sum_{w \in W} \Delta_{\alpha}\left(r_{w}\right) s_{\alpha} \Delta_{I_{v}} \Delta_{I_{w}}\left(u_{0}\right)=0
$$

for any $v \in W$. Applying the ring automorphism $s_{\alpha}$, we obtain that the $s_{\alpha} \Delta_{\alpha}\left(r_{w}\right)$ are solution of the system (1) with $x=0$ and are therefore 0 by uniqueness. Thus $\Delta_{\alpha}\left(r_{w}\right)=0$ and $r_{w}$ is fixed by $s_{\alpha}$ for any simple root $\alpha$ by Lemma 3.15 hence by the whole Weyl group $W$, since it is generated by the simple reflections.

Point (3) for $C$ is proved exactly in the same way (still using $\Delta_{\alpha}$, not $C_{\alpha}$ ).

Theorem 6.7. - Assume that the torsion index $\mathfrak{t}$ is invertible in $R$ and that $R$ has no 2-torsion. Choose a reduced decomposition $I_{w}$ for every $w \in W$. The elements $\Delta_{I_{w}}\left(u_{0}\right)$ (resp. $\left.C_{I_{w}}\left(u_{0}\right)\right)$ form a basis of $R \llbracket M \rrbracket_{F}$ as an $R \llbracket M \rrbracket_{F}^{W}$-module. 
Proof. - We need to show that any $x$ can be decomposed in a unique way as $x=\sum_{w \in W} r_{w} \Delta_{I_{w}}\left(u_{0}\right)$. Note that this is the row $v=1$ of the system (1) of Proposition 6.6. By $R \llbracket M \rrbracket_{F}^{W}$-linearity of any $\Delta_{I_{v}}$ (see Prop. 3.14), if that row is satisfied with coefficients $r_{w}$ in $R \llbracket M \rrbracket_{F}^{W}$, the rest of the system is satisfied, so this proves uniqueness. Existence of the decomposition then follows from parts (1) and (3) of the proposition. The same proof goes through with the $C_{I_{w}}\left(u_{0}\right)$.

REMARK 6.8. - The previous theorem for the (uncompleted) symmetric algebra, i.e., the additive case, is [7, $\$ 6$, Théorème 2, (c)], but the proof given there is incorrect: in the notation of loc. cit., the ideal $I$ is only known a priori to be of the right form to apply the graded Nakayama lemma when it is tensored by $\mathbb{Q}$, a fact that cannot be assumed, this is the whole point of the theorem. When contacted by one of the authors, Demazure kindly and quickly supplied another proof which we adapted to our setting in Proposition 6.6. There is a slight difference: in the symmetric algebra case, the matrix of the system is upper triangular with diagonal $t$ and is therefore invertible, whereas in our case, the lower triangular part does not vanish because our formal group law is not additive, but the strictly upper triangular part is in the radical because our ring is complete.

Theorem 6.9. - Assume that the torsion index $\mathfrak{t}$ is invertible in $R$ and that $R$ has no 2-torsion. Then the kernel of the characteristic map $\mathfrak{c}: R \llbracket M \rrbracket_{F} \rightarrow \mathcal{H}(M)_{F}$ is the ideal of $R \llbracket M \rrbracket_{F}$ generated by elements in $\mathcal{J}_{F}^{W}$.

Proof. - By Remark 6.2, the ideal generated by $J_{F}^{W}$ is included in ker c. Conversely, let $x \in$ ker $\mathfrak{c}$, and decompose it as $\sum_{w \in W} r_{w} \Delta_{I_{w}}\left(u_{0}\right)$ with the $r_{w} \in R \llbracket M \rrbracket_{F}^{W}$ by the previous theorem. We then have $0=\mathfrak{c}(x)=\sum_{w \in W} \epsilon\left(r_{w}\right) \mathfrak{c}\left(\Delta_{I_{w}}\left(u_{0}\right)\right)$. But the $\mathfrak{c}\left(\Delta_{I_{w}}\left(u_{0}\right)\right)$ form an $R$-basis of the image of $\mathfrak{c}$ by Theorem 6.4 , so $\epsilon\left(r_{w}\right)=0$ for all $w \in W$ and $x$ is the ideal generated by $\mathcal{J}_{F}^{W}$.

\section{The product structure of the cohomology}

In this section, we explain how to define a product on $\mathscr{H}(M)_{F}$ in order that the characteristic map be a ring homomorphism. Then, we study the structure of $\mathscr{H}(M)_{F}$.

Lemma 7.1. - For any $D \in \mathscr{D}(M)_{F}$,

1. there is a finite family $\left(D_{i}, D_{i}^{\prime}\right)$ of elements of $\mathscr{D}(M)_{F}$ such that $D(u v)=\sum_{i} D_{i}(u) D_{i}^{\prime}(v)$ for any elements $u, v \in R \llbracket M \rrbracket_{F}$.

2. Furthermore if $D$ is in $\mathscr{D}(M)_{F}^{(j)}$, each $D_{i}$ and $D_{i}^{\prime}$ can be chosen in $\mathscr{D}(M)_{F}^{\left(m_{i}\right)}$ and $\mathscr{D}(M)_{F}^{\left(m_{i}^{\prime}\right)}$ respectively, such that $m_{i}+m_{i}^{\prime} \geq j$.

Proof. - To show (1), the elements of $\mathscr{D}(M)_{F}$ satisfying the statement form a sub$R \llbracket M \rrbracket_{F}$-algebra of $\mathscr{D}(M)_{F}$, and it is clear for generators of $\mathscr{D}(M)_{F}$ by Equation (4) of Prop. 3.8. This same equation also proves (2) for generators by induction.

Let $R \llbracket M \rrbracket_{F}^{o \vee}$ be the continuous $R$-dual of $R \llbracket M \rrbracket_{F}$, i.e., the set of $R$-linear morphisms $f$ from $R \llbracket M \rrbracket_{F}$ to $R$ such that $f\left(\mathcal{J}_{F}^{i}\right)=0$ for some $i$ (depending on $f$ ).

4 e SÉRIE - TOME $46-2013$ - No 3 
Lemma 7.2. - The R-module $R \llbracket M \rrbracket_{F}^{o v}$ is flat. When $\mathfrak{t}$ is regular in $R$, the $R$-module $\epsilon \mathscr{D}(M)_{F}$ is also flat.

Proof. - For any $i$, the $R$-module $\mathcal{J}_{F}^{i} / \mathcal{J}_{F}^{i-1}$ is a free $R$-module since it is isomorphic to $S_{R}^{i}(M)$. Thus, by induction, for any $i$, the quotient $R \llbracket M \rrbracket_{F} / \mathcal{J}_{F}^{i}$ is a finitely generated free $R$-module, thus flat. Now $R \llbracket M \rrbracket_{F}^{o \vee}$ is the direct limit of the $\left(R \llbracket M \rrbracket_{F} / \mathcal{I}^{i}\right)^{\vee}$ (usual $R$-duals) and it is therefore flat. When $\mathfrak{t}$ is regular, the module $\epsilon \mathscr{D}(M)_{F}$ is a finitely generated free $R$-module by 5.4 , so it is flat.

TheOREm 7.3. - Let $\mathfrak{t}$ be regular in $R$. There is a unique ring structure on $\mathscr{H}(M)_{F}$ such that the characteristic map $\mathfrak{c}: R \llbracket M \rrbracket_{F} \rightarrow \mathcal{H}(M)_{F}$ is a ring morphism.

Proof. - Since $R \rightarrow R[1 / \mathfrak{t}]$ is an injection and $\mathcal{H}(M)_{F}$ is a free $R$-module, we can check uniqueness after inverting $\mathfrak{t}$, in which case it is obvious since the characteristic map is surjective. Let us now prove the existence of this product. The $R$-module $R \llbracket M \rrbracket_{F}^{o \vee}$ has a natural structure of coalgebra induced by the collection of maps

$$
\left(R \llbracket M \rrbracket_{F} / \mathcal{I}^{i}\right)^{\vee} \rightarrow\left(R \llbracket M \rrbracket_{F} / \mathcal{I}^{i}\right)^{\vee} \otimes\left(R \llbracket M \rrbracket_{F} / \mathcal{I}^{i}\right)^{\vee}
$$

dual to the product, and taking direct limits. The inclusion $\epsilon \mathscr{D}(M)_{F} \subseteq R \llbracket M \rrbracket_{F}^{o \vee}$ induces the diagonal map $\epsilon \mathscr{D}(M)_{F} \otimes_{R} \epsilon \mathscr{D}(M)_{F} \rightarrow R \llbracket M \rrbracket_{F}^{o \vee} \otimes_{R} R \llbracket M \rrbracket_{F}^{o \vee}$ which is injective by Lemma 7.2. To show that $\epsilon \mathscr{D}(M)_{F}$ is a subcoalgebra of $R \llbracket M \rrbracket_{F}^{o V}$, it therefore suffices to show that the composition $\epsilon \mathscr{D}(M)_{F} \rightarrow R \llbracket M \rrbracket_{F}^{o \vee} \rightarrow R \llbracket M \rrbracket_{F}^{o \vee} \otimes_{R} R \llbracket M \rrbracket_{F}^{o \vee}$ factors through the image of $\epsilon \mathscr{D}(M)_{F} \otimes_{R} \epsilon \mathscr{D}(M)_{F}$ in $R \llbracket M \rrbracket_{F}^{o \vee} \otimes_{R} R \llbracket M \rrbracket_{F}^{o \vee}$. This is ensured by Lemma 7.1. Now $\mathcal{H}(M)_{F}$ is the dual of a coalgebra, and is therefore an $R$-algebra. By construction, the characteristic map is a ring homomorphism.

7.4. - We now filter the ring $\mathcal{H}(M)_{F}$ by setting that $\mathcal{H}(M)_{F}^{(i)}$ is the set of forms on $\epsilon \mathscr{D}(M)_{F}$ that are zero on $\epsilon \mathscr{D}(M)_{F}^{(-i+1)}$. By Proposition 5.6, we therefore have $\mathcal{H}(M)_{F}^{(0)}=\mathscr{H}(M)_{F}$ and $\mathcal{H}(M)_{F}^{(N+1)}=0$. In other words, the filtration has the form

$$
\mathscr{H}(M)_{F}=\mathscr{H}(M)_{F}^{(0)} \supseteq \mathcal{H}(M)_{F}^{(1)} \supseteq \cdots \supseteq \mathcal{H}(M)_{F}^{(N)} \supseteq 0 .
$$

We consider the augmentation map $\varepsilon: \mathscr{H}(M)_{F} \rightarrow R$ defined as the evaluation at $\epsilon \in \epsilon \mathscr{D}(M)_{F}$.

Proposition 7.5. - Assume $\mathfrak{t}$ is regular in $R$. We have:

1. $\mathcal{H}(M)_{F}^{(1)}$ is the kernel of $\varepsilon$.

2. The filtration is compatible with the product:

$$
\mathscr{H}(M)_{F}^{(j)} \mathcal{H}(M)_{F}^{(k)} \subseteq \mathcal{H}(M)_{F}^{(j+k)} .
$$

\section{The characteristic map $\mathfrak{c}$ is a morphism of filtered rings.}

Proof. - Point (1) is clear since $\epsilon$ generates $\mathscr{D}(M)_{F}^{(0)}$. Let $h_{1} \in \mathcal{H}(M)_{F}^{(j)}$ and $h_{2} \in \mathscr{H}(M)_{F}^{(k)}$. The product $h=h_{1} . h_{2}$ is defined as $h(\epsilon D)=\sum_{i} h_{1}\left(\epsilon D_{i}\right) h_{2}\left(\epsilon D_{i}^{\prime}\right)$ where the $\left(D_{i}, D_{i}^{\prime}\right)$ are as in Lemma 7.1. By the same lemma, if $D \in \mathscr{D}(M)_{F}^{(-j-k+1)}$, they can be chosen such that $D_{i} \in \mathscr{D}(M)_{F}^{\left(j_{i}\right)}$ and $D_{i}^{\prime} \in \mathscr{D}(M)_{F}^{\left(k_{i}\right)}$ with $j_{i}+k_{i} \geq-j-k+1$. Thus, for any $i$, either $j_{i} \geq-j+1$ or $k_{i} \geq-k+1$, so every term in the sum is zero and 
$h \in \mathcal{H}(M)_{F}^{(j+k)}$. For part (3), take an element $u$ in $\mathcal{J}_{F}^{i}$. By definition, $\mathfrak{c}(u)$ is the evaluation at $u$ in $\epsilon \mathscr{D}(M)_{F}^{\vee}=\mathcal{H}(M)_{F}$ so by Prop. $5.6(1), \mathfrak{c}(u)$ is in $\mathcal{H}(M)_{F}^{(i)}$.

Proposition 7.6. - Let $I_{w}$ be choices of reduced decompositions for every $w \in W$. Assume the torsion index $\mathfrak{t}$ is regular in $R$. Then

1. For any $i$, the elements $z_{I_{w}}^{\Delta}$ (resp. $\left.z_{I_{w}}^{C}\right)$ with $l(w) \geq i$ generate $\mathcal{H}(M)_{F}^{(i)}$.

2. The elements $z_{I_{w_{0}}}^{\Delta}$ and $z_{I_{w_{0}}}^{C}$ of Theorem 6.3 do not depend on the choices of decompositions $I_{w}$ and are equal up to the sign $(-1)^{N}$. The element $z_{I_{w_{0}}}^{C}$ is denoted by $z_{0}$ and for any $u_{0}$ as in Section 5, we have $\mathfrak{c}\left(u_{0}\right)=(-1)^{N} \mathfrak{t} z_{0}$.

Proof. - Point (1) follows from part (3) of Proposition 5.6 since the $z_{I_{w}}^{\Delta}$ (resp. $z_{I_{w}}^{C}$ ) are the dual basis to the $\epsilon \Delta_{I_{w}}$ (resp. $\epsilon C_{I_{w}}$ ). By Theorem 6.3, we have $\mathfrak{c}\left(u_{0}\right)=\mathfrak{t} z_{I_{w_{0}}}^{\Delta}$. But the left hand side is independent of the choices of the $I_{w}$ and the right hand side is independent of the choice of $u_{0}$, which proves the claim. We also have $\mathfrak{c}\left(u_{0}\right)=(-1)^{N} \mathfrak{t} z_{0}$.

7.7. - We now consider operators on $\mathcal{H}(M)_{F}$. For any root $\alpha$, multiplication on the right by $C_{\alpha}$ (resp. $\left.\Delta_{\alpha}\right)$ ) defines an endomorphism on $\epsilon \mathscr{D}(M)_{F}$, and therefore one on $\mathscr{H}(M)_{F}$ by duality. This operator is denoted by $A_{\alpha}$ (resp. $B_{\alpha}$ ). In other words, for any $h \in \mathcal{H}(M)_{F}$ and $\epsilon D \in \epsilon \mathscr{D}(M)_{F}$, we have

$$
A_{\alpha}(h)(\epsilon D)=h\left(\epsilon D C_{\alpha}\right) \quad \text { and } \quad B_{\alpha}(h)(\epsilon D)=h\left(\epsilon D \Delta_{\alpha}\right) .
$$

As for the operators $\Delta$ and $C$, we use the notation $A_{I}$ and $B_{I}$ for a sequence $I$ of simple reflections.

Proposition 7.8. - When $\mathfrak{t}$ is regular in $R$, these operators satisfy:

1. $A_{\alpha} \circ \mathfrak{c}(u)=\mathfrak{c} \circ C_{\alpha}(u)$ and $B_{\alpha} \circ \mathfrak{c}(u)=\mathfrak{c} \circ \Delta_{\alpha}(u)$.

2. $A_{\alpha} \mathcal{H}(M)_{F}^{(i)} \subseteq \mathcal{H}(M)_{F}^{(i-1)}$ and $B_{\alpha} \mathcal{H}(M)_{F}^{(i)} \subseteq \mathcal{H}(M)_{F}^{(i-1)}$.

3. For any reduced decomposition $I_{0}$ of $w_{0}$, the element $A_{I_{0}}\left(z_{0}\right)$ (resp. $B_{I_{0}}\left(z_{0}\right)$ ) is invertible in $\mathcal{H}(M)_{F}$.

4. If $I$ is nonreduced and of length $N$, then the element $A_{I}\left(z_{0}\right)$ (resp. $\left.B_{I}\left(z_{0}\right)\right)$ is in $\mathcal{H}(M)_{F}^{(1)}=\operatorname{ker} \varepsilon$.

Proof. - Point (1) follows directly from the definitions of the characteristic map and of the operators. Point (2) follows from part (1) of Proposition 4.6. For (3), first note that by Proposition 7.5, an element $h \in \mathcal{H}(M)_{F}$ is invertible if and only if $\varepsilon(h)$ is invertible in $R$.

Now

$$
\mathfrak{t} \varepsilon A_{I_{0}}\left(z_{0}\right)=\varepsilon A_{I_{0}}\left(\mathfrak{t} z_{0}\right)=\varepsilon A_{I_{0}} \mathfrak{c}\left(u_{0}\right)=\varepsilon \mathfrak{c}\left(C_{I_{0}}\left(u_{0}\right)\right)=\epsilon C_{I_{0}}\left(u_{0}\right)=\mathfrak{t} .
$$

Therefore, $\varepsilon A_{I_{0}}\left(z_{0}\right)=1$. The same proof works for $\varepsilon B_{I_{0}}\left(z_{0}\right)$. For part (4), the same series of equalities is used, except that the last term is zero by Prop. 5.3.

Proposition 7.9. - Let $I_{w}$ be a choice of reduced decompositions for all $w \in W$. Assume $\mathfrak{t}$ is regular in $R$. Then the elements $A_{I_{w}}\left(z_{0}\right)$ (resp. $B_{I_{w}}\left(z_{0}\right)$ ) with $l(w) \leq N-i$ form an $R$-basis of $\mathcal{H}(M)_{F}^{(i)}$. 
Proof. - We prove it for the $A_{I_{w}}\left(z_{0}\right)$, the proof for the $B_{I_{w}}\left(z_{0}\right)$ is similar. First note that by Proposition 7.6, $z_{0}$ is the unique element of $\mathcal{H}(M)_{F}$ such that $z_{0}\left(\epsilon C_{I_{w}}\right)=\delta_{w, w_{0}}$. Thus, if $l(w)+l(v) \leq N$, we have $A_{I_{w}}\left(z_{0}\right)\left(\epsilon C_{I_{v}}\right)=\delta_{w, v^{-1} w_{0}}$. In other words, if we decompose $A_{I_{w}}$ on the basis of the $z_{I_{v}}$, the coordinate on $z_{I_{v}}$ is $\delta_{w, v^{-1} w_{0}}$ if $l(v)+l(w) \leq N$. Thus, the $A_{I_{w}}\left(z_{0}\right)$ with $l(w) \leq N-i$ can be expressed as linear combinations of the $z_{I_{v}}$ with $l(v) \geq i$ and the matrix of their expressions is unitriangular up to a correct ordering of rows and columns and is therefore invertible.

Proposition 7.10. - Assume $\mathfrak{t}$ is regular in $R$ and $R^{\prime}$, respectively endowed with formal group laws $F$ and $F^{\prime}$. Let $f: R \rightarrow R^{\prime}$ be a ring morphism sending $F$ to $F^{\prime}$. Then the morphism $f_{\mathscr{H}}: \mathcal{H}_{R}(M)_{F} \rightarrow \mathcal{H}_{R^{\prime}}(M)_{F^{\prime}}$ introduced before Proposition 6.5 is actually a morphism of filtered rings. It satisfies

$$
A_{I}^{F^{\prime}} f_{\mathscr{H}}=f_{\mathscr{H}} A_{I}^{F} \quad \text { and } \quad B_{I}^{F^{\prime}} f_{\mathscr{H}}=f_{\mathscr{H}} B_{I}^{F}
$$

for any sequence $I$.

Proof. - Both facts are clear by Point 2 of 6.5 when the characteristic map is surjective, and therefore when $\mathfrak{t}$ is regular by extending scalars to $R[1 / t]$.

\section{PART II \\ BOTT-SAMELSON RESOLUTIONS FOR ORIENTED THEORIES}

\section{Algebraic cobordism and oriented cohomology theories}

In the present section we recall the notion of oriented cohomology theory and the notion of algebraic cobordism $\Omega$ following the book of Levine and Morel [14]. As main examples of oriented theories we consider the Chow ring $\mathrm{CH}$, Grothendieck's $\mathrm{K}^{0}$ and connective $K$-theory k.

8.1. - According to $[14, \S 1.1]$ an oriented cohomology theory $\mathrm{h}$ is a contravariant functor from the category of smooth varieties over a field $k$ of an arbitrary characteristic to the category of graded rings satisfying the standard cohomological axioms: existence of pushforwards for projective morphisms, the projection formula, base change, homotopy invariance and the projective bundle axiom (see [14, Def. 1.1.2]).

A universal theory $\Omega$ satisfying these properties was constructed in $[14, \S 2]$ assuming the base field $k$ has characteristic 0 . It is called algebraic cobordism. An element of codimension $i$ in $\Omega(X)$, i.e., in $\Omega^{i}(X)$, is additively generated by classes $[Y \rightarrow X]$ of projective maps of codimension $i$ from smooth schemes $Y$.

For any map $f: X_{1} \rightarrow X_{2}$ the induced functorial map $f^{*}: \Omega^{i}\left(X_{2}\right) \rightarrow \Omega^{i}\left(X_{1}\right)$ is called the pull-back. For a projective map $f: X_{1} \rightarrow X_{2}$, there is a corresponding push-forward map $f_{*}: \Omega\left(X_{1}\right) \rightarrow \Omega\left(X_{2}\right)$ shifting the cohomological degree by $\operatorname{dim} X_{2}-\operatorname{dim} X_{1}$ when $X_{1}$ and $X_{2}$ are equidimensional and given by $\left[Y_{1} \rightarrow X_{1}\right] \mapsto\left[Y_{1} \rightarrow X_{1} \stackrel{f}{\rightarrow} X_{2}\right]$. 
8.2. - An oriented cohomology theory h comes with a formal group law $F$ over the coefficient ring $\mathrm{h}(\mathrm{pt})$ such that

$$
F\left(c_{1}^{\mathrm{h}}\left(\mathscr{L}_{1}\right), c_{2}^{\mathrm{h}}\left(\mathscr{L}_{2}\right)\right)=c_{1}^{\mathrm{h}}\left(\mathscr{L}_{1} \otimes \mathscr{L}_{2}\right),
$$

where $\mathscr{L}_{1}$ and $\mathscr{L}_{2}$ are lines bundles on $X$ and $c_{1}^{\mathrm{h}}$ denotes the first Chern class in the cohomology theory h (see [14, Cor.4.1.8]). For $\Omega$, the associated formal group law $U$

$$
U(x, y)=x+y+\sum_{i, j \geq 1} a_{i j} x^{i} y^{j}, \quad \text { where } a_{i j} \in \Omega(\mathrm{pt})
$$

turns out to be universal. The ring of coefficients $\Omega(\mathrm{pt})$ is generated by the coefficients $a_{i j}$ and coincides with the classical Lazard ring $\mathbb{L}$.

There is a canonical map $p r_{\mathrm{h}}: \Omega \rightarrow \mathrm{h}$ sending the coefficients of $U$ to the corresponding coefficients of $F$, and hence inducing a morphism of formal group laws.

Example 8.3. - Consider the Chow ring $\mathrm{CH}(X)$ of algebraic cycles on $X$ modulo rational equivalence. According to [14, Thm. 4.5.1] the canonical map $p r_{\mathrm{CH}}: \Omega \rightarrow \mathrm{CH}$ induces an isomorphism $\Omega \otimes_{\mathbb{L}} \mathbb{Z} \stackrel{\simeq}{\rightarrow} \mathrm{CH}$ of oriented cohomology theories. In particular, $p r_{\mathrm{CH}}: \Omega \rightarrow \mathrm{CH}$ is surjective and its kernel is generated by $\mathbb{L}_{>0}$, the subgroup of elements of positive dimension in the Lazard ring. Observe that $p r_{\mathrm{CH}}$ restricted to the coefficient rings $\mathbb{L} \rightarrow \mathbb{Z}=\mathrm{CH}(\mathrm{pt})$ coincides with the augmentation map. The associated formal group law, denoted by $F_{0}$, is called the additive formal group law and is given by $F_{0}(x, y)=x+y$.

EXAMPLE 8.4. - Consider the oriented cohomology theory $\mathrm{K}(X)=\mathrm{K}^{0}(X)\left[\beta, \beta^{-1}\right]$, where $\mathrm{K}^{0}(X)$ is the Grothendieck $\mathrm{K}^{0}$ of $X$. Observe that $\mathrm{K}(\mathrm{pt})=\mathbb{Z}\left[\beta, \beta^{-1}\right]$. According to [14, Cor.4.2.12] the canonical map $p r_{\mathrm{K}}: \Omega \rightarrow \mathrm{K}$ sending $\left[\mathbb{P}^{1}\right]$ to $\beta$ induces an isomorphism $\Omega \otimes_{\mathbb{L}} \mathbb{Z}\left[\beta, \beta^{-1}\right] \rightarrow \mathrm{K}$ of oriented cohomology theories. The associated formal group law, denoted by $F_{\beta}$, is called a multiplicative periodic formal group law and is given by $F_{\beta}(x, y)=x+y-\beta x y$.

EXAmple 8.5. - Consider a ring homomorphism $\mathbb{L} \rightarrow \mathbb{Z}[v]$ given by $\left[\mathbb{P}^{1}\right]=-a_{11} \mapsto v$ and $a_{i j} \mapsto 0$ for $(i, j) \neq(1,1)$. We define a new cohomology theory, called a connective $K$-theory, by $\mathrm{k}=\Omega \otimes_{\mathbb{L}} \mathbb{Z}[v]$ (see [14, §4.3.3]). Its formal group law is denoted by $F_{v}$ and is given by $F_{v}(x, y)=x+y-v x y$. Observe that contrary to Example 8.4 the element $v$ is non-invertible in the coefficient ring $\mathrm{k}(\mathrm{pt})=\mathbb{Z}[v]$.

REMARK 8.6. - In positive characteristic, one can replace $\Omega$ by the theory $\widetilde{\mathrm{CH}}$ obtained by applying Merkurjev's "tilde operation" [15] to Chow theory. Note that the Lazard ring $\mathbb{L}$ injects into the coefficient ring $\tilde{\mathrm{CH}}(\mathrm{pt})$.

Let us prove a general fact concerning cellular filtrations.

Definition 8.7. - The oriented cohomology theory $h$ is said to satisfy the weak localization axiom if for any closed embedding $D \rightarrow X$ between smooth schemes with open complement $U$, the sequence $\mathrm{h}(Z) \rightarrow \mathrm{h}(X) \rightarrow \mathrm{h}(U)$, composed of the push-forward from $D$ followed by the restriction to $U$, is exact. 
Let now $X$ be a smooth variety over $k$ together with a filtration

$$
U_{0}=\varnothing \subset U_{1} \subset \cdots \subset U_{n}=X
$$

by open subsets, such that for each $i=1, \ldots, n$, the closed complement $A_{i}=U_{i} \backslash U_{i-1}$ has a scheme structure of affine spaces over $k$. Let $Z_{i}$ be the closure of $A_{i}$ in $X$. Note in particular that all $U_{i}$ are smooth varieties over $k$, and that $Z_{i} \cap U_{i}=A_{i}$. Let $X_{i}$ be a desingularization of $Z_{i}$ by a projective morphism, such that the preimage of $U_{i}$ in $X_{i}$ maps isomorphically to $U_{i}$ (this is not really needed, but it simplifies exposition, and will be true in the cases where we will use this setting). Let $\zeta_{i}$ be the pushforward of $1_{Z_{i}} \in \mathrm{h}\left(Z_{i}\right)$ to $\mathrm{h}(X)$.

THEOREM 8.8. - If $\mathrm{h}$ satisfies the weak localization axiom, then the classes $\zeta_{i}$ generate $\mathrm{h}(X)$ as a module over $\mathrm{h}(\mathrm{pt})$.

Proof. - By base change, the class $\zeta_{i}$ restricted to $U_{i}$ is the push-forward of $1_{A_{i}} \in \mathrm{h}\left(A_{i}\right)$ to $\mathrm{h}\left(U_{i}\right)$. By homotopy invariance, $\mathrm{h}\left(A_{i}\right)$ is a free $\mathrm{h}(\mathrm{pt})$-module of rank 1 and $1_{A_{i}}$ is a basis. Now, by induction, using exactness (in the middle only) of the localization sequence $\mathrm{h}\left(A_{i}\right) \rightarrow \mathrm{h}\left(U_{i}\right) \rightarrow \mathrm{h}\left(U_{i-1}\right)$, one simultaneously proves the following facts: 1 . The restriction from $\mathrm{h}\left(U_{i}\right)$ to $\mathrm{h}\left(U_{i-1}\right)$ is surjective. 2. $\mathrm{h}\left(U_{i}\right)$ is generated by the restrictions of the classes $\zeta_{k}$ for $k \leq i$.

We now come to the following important property of an algebraic cohomology theory:

Definition 8.9. - We say that a cohomology theory is weakly birationally invariant if for any proper birational morphism $f: Y \rightarrow X$ the push-forward of the fundamental class $f_{*}\left(1_{Y}\right)$ is invertible.

EXAmPLE 8.10. - Any birationally invariant theory, i.e., such that $f_{*}\left(1_{Y}\right)=1_{X}$ for any proper birational $f: Y \rightarrow X$, is weakly birationally invariant. The Chow ring $\mathrm{CH}$ considered over an arbitrary field and the $K$-theory $\mathrm{K}$ considered over a field of characteristic 0 provide examples of birationally invariant theories. According to [14, Thm.4.3.9] the connective $K$-theory $\mathrm{k}$ defined over a field of characteristic 0 is universal among all birationally invariant theories. In particular, the kernel of the canonical map $\mathbb{L} \rightarrow \mathrm{k}(\mathrm{pt})$ is generated by differences of classes $[X]-\left[X^{\prime}\right]$, where $X$ and $X^{\prime}$ are birationally equivalent.

Lemma 8.11. - For any smooth variety $X$ over a field of characteristic 0 , the kernel of the natural map $\Omega^{n}(X) \rightarrow \mathrm{CH}^{n}(X)$ consists of nilpotent elements if $n \geq 0$ and is trivial if $n=\operatorname{dim} X$.

Proof. - According to [14, Rem. 4.5.6] the kernel is additively generated by products of the form $a b, a \in \mathbb{L}^{-i}, b \in \Omega^{n+i}(X), i>0$. Now the claim follows from the fact that $\Omega^{j}(X)=0$ when $j>\operatorname{dim} X$.

COROLLARY 8.12. - Over a field of characteristic 0 , any oriented cohomology theory in the sense of Levine-Morel is weakly birationally invariant.

Proof. - Consider the algebraic cobordism $\Omega$. Let $f: Y \rightarrow X$ be a proper birational map. The element $f_{*}^{\Omega}\left(1_{Y}\right)-1_{X}$ is in the kernel of the map $\Omega^{0}(X) \rightarrow \mathrm{CH}^{0}(X)$. By Lemma 8.11 the difference $f_{*}^{\Omega}\left(1_{Y}\right)-1_{X}$ is nilpotent, therefore, $f_{*}^{\Omega}\left(1_{Y}\right)$ is invertible. The statement for an arbitrary theory follows by the universality of $\Omega$. 


\section{A sequence of split $\mathbb{P}^{1}$-bundles}

In the present section we compute the oriented cohomology $h$ of a variety obtained as a sequence of split $\mathbb{P}^{1}$-bundles. The main tool is the projective bundle theorem for $h$. Observe that all formulas are given in terms of pull-backs and push-forwards of fundamental classes. This invariant description will play an important role in the sequel.

Lemma 9.1. - Let $X$ be a smooth projective variety over a field $k$. Let $p: \mathbb{P}_{X}(\mathcal{E}) \rightarrow X$ be the projective bundle of a vector bundle $\mathcal{E}$ of rank 2 over $X$. Assume that $p$ has a section $\sigma$. Then there is a ring isomorphism

$$
\mathrm{h}\left(\mathbb{P}_{X}(\mathcal{E})\right) \simeq \mathrm{h}(X)[\xi] /\left(\xi^{2}-y \xi\right), \text { where } \xi=\sigma_{*}\left(1_{X}\right) \text { and } y=p^{*} \sigma^{*} \xi .
$$

Proof. - Consider the canonical embedding $\theta_{\mathcal{E}}(-1) \rightarrow p^{*} \mathcal{E}$, where $\theta_{\mathcal{E}}(-1)$ is a tautological line bundle over $\mathbb{P}_{X}(\mathcal{E})$ (see [9, B.5.5]). Let $\mathscr{L}$ denote the quotient $\mathcal{E} / \sigma^{*} \theta_{\mathcal{E}}(-1)$. By the Whitney formula (see [14, Prop. 4.1.15]) we have

$$
c_{1}^{\mathrm{h}}(\mathscr{E})=c_{1}^{\mathrm{h}}\left(\sigma^{*} \vartheta_{\mathscr{E}}(-1)\right)+c_{1}^{\mathrm{h}}(\mathscr{L}) \quad \text { and } \quad c_{2}^{\mathrm{h}}(\mathcal{E})=c_{1}^{\mathrm{h}}\left(\sigma^{*} \theta_{\mathscr{E}}(-1)\right) \cdot c_{1}^{\mathrm{h}}(\mathscr{L}) .
$$

According to the projective bundle theorem (see [14, Def. 1.1.2 and Chern classes on p.3]) applied to $p$, there is a ring isomorphism

$$
\mathrm{h}\left(\mathbb{P}_{X}(\mathcal{E})\right) \simeq \mathrm{h}(X)[t] /(t-a)(t-b),
$$

where $a=p^{*} c_{1}^{\mathrm{h}}\left(\sigma^{*} \Theta_{\mathcal{E}}(-1)\right), b=p^{*} c_{1}^{\mathrm{h}}(\mathscr{L})$ and $t=c_{1}^{\mathrm{h}}\left(\Theta_{\mathcal{E}}(-1)\right)$.

Consider the elements $\xi=b{ }_{F} t$ and $y=b-{ }_{F} a$, where $F$ is the formal group law corresponding to h. Observe that by $[17,2.2 .9] \xi=c_{1}^{\mathrm{h}}\left(\theta_{\mathcal{E}}(1) \otimes p^{*} \mathscr{L}\right)=\sigma_{*}\left(1_{X}\right)$ and by the very definition $y=c_{1}^{\mathrm{h}}\left(p^{*} \sigma^{*} \theta_{\mathscr{E}}(1) \otimes p^{*} \mathscr{L}\right)=p^{*} \sigma^{*} \xi$.

Since changing $t$ to $\xi$ induces an automorphism of $h\left(\mathbb{P}_{X}(\mathscr{E})\right)$, we only need to prove the relation $\xi^{2}-y \xi=0$. Note that for any power series $f$, we may write $f(x)-f(y)=$ $(x-y) f^{\prime}(x, y)$ where $f^{\prime}(x, y)$ is again a power series. Hence, we have $b-{ }_{F} t=$ $\left(b-{ }_{F} t\right)-\left(t-{ }_{F} t\right)=(t-b) f_{1}^{\prime}(b, t)$ and $\left(b-{ }_{F} t\right)-\left(b-{ }_{F} a\right)=(t-a) f_{2}^{\prime}(a, b, t)$ for some power series $f_{1}^{\prime}$ and $f_{2}^{\prime}$. Therefore

$$
\xi(\xi-y)=\left(b-{ }_{F} t\right)\left(\left(b-{ }_{F} t\right)-\left(b-{ }_{F} a\right)\right)=(t-b)(t-a) f_{1}^{\prime}(b, t) f_{2}^{\prime}(a, b, t)=0
$$

and the proof is finished.

COROLlary 9.2. - In the notation of Lemma 9.1 we have the following formula for the push-forward of the fundamental class

$$
p_{*}\left(1_{\mathbb{P}_{X}(\xi)}\right)=\sigma^{*}\left(\xi^{-1}+\left(-{ }_{F} \xi\right)^{-1}\right) .
$$

Proof. - By [22, Thm. 5.30] we have

$$
p_{*}\left(1_{\mathbb{P}_{X}(\delta)}\right)=\left(\sigma^{*} b-{ }_{F} \sigma^{*} a\right)^{-1}+\left(\sigma^{*} a-{ }_{F} \sigma^{*} b\right)^{-1},
$$

where $\sigma^{*} a$ and $\sigma^{*} b$ are the roots of the bundle $\mathcal{E}$ as in the proof of Lemma 9.1. Since $y=b-{ }_{F} a=p^{*} \sigma^{*} \xi$ we obtain the desired formula. 
THEOREM 9.3. - More generally, let $X$ be a variety obtained by means of a sequence of split $\mathbb{P}^{1}$-bundles, i.e., there is a sequence of varieties $X_{i}, 0 \leq i \leq N$, starting from a point $X_{0}=\mathrm{pt}$ and finishing at $X_{N}=X$ such that for each $1 \leq i \leq N$

$$
p_{i}: X_{i} \simeq \mathbb{P}_{X_{i-1}}\left(\mathcal{E}_{i}\right) \rightarrow X_{i-1}
$$

is a projective bundle with a section $\sigma_{i}$, where $\mathcal{E}_{i}$ is some vector bundle of rank 2 over $X_{i-1}$. Then there is a ring isomorphism

$$
\mathrm{h}\left(X_{N}\right) \simeq \mathrm{h}(\mathrm{pt})\left[\xi_{1}, \ldots, \xi_{N}\right] / I
$$

where $I$ is an ideal generated by elements $\left\{\xi_{i}^{2}-y_{i} \xi_{i}\right\}_{i=1 \ldots N}, \xi_{i}=p^{*} \sigma_{i *}\left(1_{X_{i-1}}\right)$, $y_{i}=p^{*} \sigma_{i}^{*} \sigma_{i *}\left(1_{X_{i-1}}\right)$ and $p^{*}$ denotes the pull-back on $X_{N}$.

Proof. - Follows by induction using Lemma 9.1.

REMARK 9.4. - Observe that the element $y_{i}$ appearing in the relations is not necessarily in $\mathrm{h}(\mathrm{pt})$. To obtain a complete answer in terms of generators $\xi_{1}, \ldots, \xi_{N}$ and relations with coefficients in $\mathrm{h}(\mathrm{pt})$ one has to express the elements $y_{i}$ in terms of the 'previous' generators $\xi_{1}, \ldots, \xi_{i-1}$. In the next section, we show how to obtain such an expression for BottSamelson varieties using the characteristic map.

Example 9.5. - Let $X$ be as in Lemma 9.1. By [9, 3.2.11] we have the following formula for the total Chern class of the tangent bundle of $\mathbb{P}_{X}(\varepsilon)$ :

$$
c^{\mathrm{h}}\left(\mathcal{J}_{\mathbb{P}_{X}(\mathcal{E})}\right)=c^{\mathrm{h}}\left(p^{*} \mathcal{T}_{X}\right) \cdot c^{\mathrm{h}}\left(\vartheta_{\mathcal{E}}(1) \otimes p^{*} \mathcal{E}\right) .
$$

Since there is an exact sequence

$$
0 \rightarrow \vartheta_{\mathcal{E}}(1) \otimes p^{*} \sigma^{*} \vartheta_{\mathcal{E}}(-1) \rightarrow \vartheta_{\mathcal{E}}(1) \otimes p^{*} \mathcal{E} \rightarrow \vartheta_{\mathcal{E}}(1) \otimes p^{*} \mathscr{L} \rightarrow 0,
$$

by Cartan formula we obtain

$$
c^{\mathrm{h}}\left(\Theta_{\mathscr{E}}(1) \otimes p^{*} \mathcal{E}\right)=c^{\mathrm{h}}\left(\Theta_{\mathscr{E}}(1) \otimes p^{*} \mathscr{L}\right) \cdot c^{\mathrm{h}}\left(\Theta_{\mathscr{E}}(1) \otimes p^{*} \sigma^{*} \Theta_{\mathscr{E}}(-1)\right) .
$$

Since $\theta_{\mathcal{E}}(1) \otimes p^{*} \sigma^{*} \theta_{\mathcal{E}}(-1)=\left(\theta_{\mathcal{E}}(1) \otimes p^{*} \mathscr{L}\right) \otimes\left(p^{*} \sigma^{*} \theta_{\mathcal{E}}(-1) \otimes p^{*} \mathcal{L}^{\vee}\right)$, we obtain that

$$
c^{\mathrm{h}}\left(\mathcal{J}_{\mathbb{P}_{X}(\mathscr{\xi})}\right)=c^{\mathrm{h}}\left(p^{*} \mathcal{J}_{X}\right) \cdot(1+\xi) \cdot\left(1+\left(\xi-{ }_{F} y\right)\right) .
$$

More generally, for $X_{N}$ from Theorem 9.3 we obtain by induction

$$
c^{\mathrm{h}}\left(\mathcal{T}_{X_{N}}\right)=\prod_{i=1}^{N}\left(1+\xi_{i}\right)\left(1+\left(\xi_{i}-{ }_{F} y_{i}\right)\right) .
$$




\section{An elementary step of the Bott-Samelson resolution}

We apply the results of the previous section to compute an oriented cohomology of a split $P / B=\mathbb{P}^{1}$-bundle, where $P$ is a semi-direct product of a split reductive linear algebraic group of semisimple rank one and a connected unipotent group, and $B$ is a Borel subgroup of $P$. This example appears as an elementary step in the construction of a Bott-Samelson variety.

10.1. - Consider a split connected solvable algebraic group $B$ over a field $k$. Let $T$ denote its split maximal torus. Let $X$ be a scheme on which $B$ acts on the right such that the quotient $X / B$ exists and $X \rightarrow X / B$ is a principal $B$-bundle. Let $M$ denote the group of characters of $T$. Each character $\lambda \in M$ extends to a one-dimensional representation $V_{\lambda}$ of $B$ and, hence, defines a line bundle $\mathcal{L}(\lambda)$ over $X / B$ whose total space is the fiber product $X \times{ }^{B} V_{\lambda}$, i.e., the quotient $\left(X \times_{k} V_{\lambda}\right) / B$ by means of the right $B$-action $(x, v) b=\left(x b, b^{-1} v\right)$ (see [1, Ch.1 §3]).

Definition 10.2. - Let $\mathrm{h}$ be an oriented cohomology theory and let $F$ be the corresponding formal group law over the coefficient ring $R=\mathrm{h}(\mathrm{pt})$. We define a ring homomorphism $\mathfrak{c}: R \llbracket M \rrbracket_{F} \rightarrow \mathrm{h}(X / B)$ from the formal group ring $R \llbracket M \rrbracket_{F}$ to the cohomology ring $\mathrm{h}(X / B)$ by sending a generator $x_{\lambda}$ to the first Chern class $c_{1}^{\mathrm{h}}(\mathscr{L}(\lambda))$ of the line bundle $\mathscr{L}(\lambda)$. This map is well-defined since all Chern classes are nilpotent and satisfy $c_{1}^{\mathrm{h}}(\mathscr{L}(\lambda+\mu))=c_{1}^{\mathrm{h}}(\mathscr{L}(\lambda) \otimes \mathscr{L}(\mu))=c_{1}^{\mathrm{h}}(\mathscr{L}(\lambda))+{ }_{F} c_{1}^{\mathrm{h}}(\mathscr{L}(\mu))$. It is called the characteristic map.

10.3. - We follow the notation of [7, §2]: Let $P$ be a semi-direct product of a connected unipotent group $U$ and a reductive split group $L$ of semi-simple rank 1 . Let $T$ be a maximal split torus of $L$ and let $M$ denote its group of characters. Let $\alpha$ be the one of two roots of $L$ with respect to $T$, let $U_{\alpha}$ be the corresponding unipotent subgroup and let $B=T \cdot U_{\alpha} \cdot U$ be the Borel subgroup of $P$ containing $T$. Let $s_{\alpha}(\lambda)=\lambda-\alpha^{\vee}(\lambda) \cdot \alpha$ denote the reflection corresponding to the root $\alpha$.

Consider the fibered product $X^{\prime}=X \times{ }^{B} P$, i.e., the quotient $\left(X \times_{k} P\right) / B$ by means of the $B$-action $(x, h) b=\left(x b, b^{-1} h\right)$. By definition, $X^{\prime}$ is a principal $P$-bundle over $X / B$, all fibers of the canonical projection $p: X^{\prime} / B \rightarrow X / B$ are isomorphic to $P / B \simeq \mathbb{P}^{1}$ and there is an obvious section $\sigma: X / B \rightarrow X^{\prime} / B$ given by $x \mapsto(x, 1)$. According to [11, Exerc. 7.10.(c)] there exists a vector bundle $\mathscr{E}$ of rank 2 over $X / B$ such that $X^{\prime} / B$ can be identified with the projective bundle $\mathbb{P}_{X / B}(\mathcal{E})$.

REMARK 10.4. - Observe that in [7, §2] instead of the quotient $X^{\prime} / B$ the author considers the quotient of $X^{\prime}$ modulo the opposite Borel subgroup $B^{\prime}$. This does not change much, since there is an obvious isomorphism $i: X^{\prime} / B \rightarrow X^{\prime} / B^{\prime}$ induced by an automorphism of $X^{\prime}$ sending the class of $(x, p)$ to the class of $\left(x, p n_{\alpha}\right)$, where $n_{\alpha}$ is an element of $P$ representing the reflection $s_{\alpha}$. In particular, for any character $\lambda$ we have $i^{*} \mathscr{L}^{\prime}(\lambda)=\mathscr{L}^{\prime}\left(s_{\alpha}(\lambda)\right)$, where $\mathscr{L}^{\prime}(\lambda)$ (resp. $\mathscr{L}^{\prime}\left(s_{\alpha}(\lambda)\right)$ ) is the line bundle over $X^{\prime} / B^{\prime}$ (resp. over $X^{\prime} / B$ ).

10.5. - Applying Lemma 9.1 to the projective bundle $p: \mathbb{P}_{X / B}(\mathcal{E}) \rightarrow X / B$ with the section $\sigma$ we obtain an isomorphism

$$
\mathrm{h}\left(X^{\prime} / B\right) \simeq \mathrm{h}(X / B)[\xi] /\left(\xi^{2}-y \xi\right), \quad \text { where } \xi=\sigma_{*}\left(1_{X / B}\right) \text { and } y=p^{*} \sigma^{*} \xi .
$$


Lemma 10.6. - We have $\sigma^{*} \xi=c_{1}^{\mathrm{h}}(\mathscr{L}(-\alpha))=\mathfrak{c}\left(x_{-\alpha}\right)$.

Proof. - By definition of the push-forward we have $\xi=c_{1}^{\mathrm{h}}(\theta(D))$, where $\theta(D)$ is a line bundle corresponding to the divisor $D=\sigma(X / B)$. The first equality then follows from [8, $\S 2.5$ Lem. 3]. The second one follows from the definition of the characteristic map $\mathfrak{c}$.

Corollary 10.7. - We have $y=p^{*} \sigma^{*} \xi=p^{*} \mathfrak{c}\left(x_{-\alpha}\right)$.

Lemma 10.8. - Consider the characteristic map $\mathfrak{c}^{\prime}: R \llbracket M \rrbracket_{F} \rightarrow \mathrm{h}\left(X^{\prime} / B\right)$. For any $u \in R \llbracket M \rrbracket_{F}$, we have

$$
\text { (1) } \sigma^{*} \mathfrak{c}^{\prime}(u)=\mathfrak{c}(u) \quad \text { and } \quad(2) \mathfrak{c}^{\prime}(u)=p^{*} \mathfrak{c}\left(s_{\alpha}(u)\right)+p^{*} \mathfrak{c}\left(\Delta_{-\alpha}(u)\right) \cdot \xi
$$

Proof. - By [8, 2.5 Lem. 1 and Prop.1], for any character $\lambda \in M$, we have two equalities

$$
\sigma^{*} \mathscr{L}^{\prime}(\lambda)=\mathscr{L}(\lambda) \quad \text { and } \quad \mathscr{L}^{\prime}\left(s_{\alpha}(\lambda)\right)=p^{*}(\mathscr{L}(\lambda)) \otimes \theta(D)^{\otimes \alpha^{\vee}(\lambda)},
$$

where $\mathcal{L}^{\prime}(\lambda)$ is the corresponding line bundle over $X^{\prime} / B$ and $\Theta(D)$ is a line bundle corresponding to the divisor $D=\sigma(X / B)$.

By the first equality, formula (1) holds for $u=x_{\lambda}$. It therefore holds for all $u \in R[M]_{F}$ and then for all $u \in R \llbracket M \rrbracket_{F}$ by continuity.

By the second equality, we have for $u=x_{\lambda}$

$$
\mathfrak{c}^{\prime}\left(x_{s_{\alpha}(\lambda)}\right)=p^{*} \mathfrak{c}\left(x_{\lambda}\right)+{ }_{F}\left(\alpha^{\vee}(\lambda)\right) \cdot_{F} \xi .
$$

Note that for any power series $g(\xi)$ in a variable $\xi$ we may write $g(\xi)=g(0)+\xi \cdot g^{\prime}(\xi)$, where $g^{\prime}(\xi)=(g(\xi)-g(0)) / \xi$ is again a power series. Since, $\xi^{2}=y \xi$, we have $g(\xi)=g(0)+\xi \cdot g^{\prime}(y)$. Applying this to $g(\xi)=p^{*} \mathfrak{c}\left(x_{\lambda}\right)+{ }_{F} \alpha^{\vee}(\lambda) \cdot{ }_{F} \xi$ and observing that $g(0)=p^{*} \mathfrak{c}\left(x_{\lambda}\right)$ we obtain

$$
\mathfrak{c}^{\prime}\left(x_{s_{\alpha(\lambda)}}\right)=p^{*} \mathfrak{c}\left(x_{\lambda}\right)+\xi \cdot g^{\prime}(y),
$$

where $g(y)=p^{*} \mathfrak{c}\left(x_{\lambda}+{ }_{F} \alpha^{\vee}(\lambda) \cdot_{F} x_{-\alpha}\right)=p^{*} \mathfrak{c}\left(x_{s_{\alpha}(\lambda)}\right)$ by Cor. 10.7. Then by definition of the operator $\Delta_{-\alpha}$ we obtain $g^{\prime}(y)=p^{*}\left(-\Delta_{-\alpha}\left(x_{\lambda}\right)\right)$ and, hence, $\mathfrak{c}^{\prime}\left(x_{s_{\alpha(\lambda)}}\right)=$ $p^{*} \mathfrak{c}\left(x_{\lambda}\right)-p^{*} \mathfrak{c}\left(\Delta_{-\alpha}\left(x_{\lambda}\right)\right) \xi$. Since $\Delta_{-\alpha} s_{\alpha}=-\Delta_{-\alpha}$, applying $s_{\alpha}$ to $u$ we obtain

$$
\mathfrak{c}^{\prime}\left(x_{\lambda}\right)=p^{*} \mathfrak{c}\left(s_{\alpha}\left(x_{\lambda}\right)\right)+p^{*} \mathfrak{c}\left(\Delta_{-\alpha}\left(x_{\lambda}\right)\right) \cdot \xi
$$

For general $u$ formula (2) then follows by induction on the degree of monomials and by continuity, using Prop. 3.8 (4) and $\xi^{2}=y \xi$.

REMARK 10.9. - Following Remark 3.7, the second formula would look simpler with the other definition of $\Delta_{\alpha}$.

Proposition 10.10. - The following formulas hold for any $u \in R \llbracket M \rrbracket_{F}$

$$
\begin{aligned}
& \text { (1) } p_{*}\left(1_{X^{\prime} / B}\right)=\mathfrak{c}\left(C_{\alpha}(1)\right),(3) \quad p^{*}\left(\mathfrak{c}\left(C_{\alpha}(u)\right)\right)=\mathfrak{c}^{\prime}\left(C_{\alpha}(u)\right) \text {, } \\
& \text { (2) } p_{*}\left(\mathfrak{c}^{\prime}(u)\right)=\mathfrak{c}\left(C_{\alpha}(u)\right), \quad \text { (4) } p^{*} p_{*}\left(\mathfrak{c}^{\prime}(u)\right)=\mathfrak{c}^{\prime}\left(C_{\alpha}(u)\right) \text {. }
\end{aligned}
$$


Proof. - The first formula of the proposition follows from Corollary 9.2 applied to the projective bundle $p: X^{\prime} / B \rightarrow X / B$ where we identify $\sigma^{*} \xi$ with $\mathfrak{c}\left(x_{-\alpha}\right)$ according to Lemma 10.6.

By (2) of Lemma 10.8 and the projection formula we have

$$
p_{*}\left(\mathfrak{c}^{\prime}(u)\right)=\mathfrak{c}\left(s_{\alpha}(u)\right) \cdot p_{*}\left(1_{X^{\prime} / B}\right)+\mathfrak{c}\left(\Delta_{-\alpha}(u)\right) \cdot p_{*}(\xi) .
$$

By the first formula and the fact that $p_{*}(\xi)=p_{*}\left(\sigma_{*}\left(1_{X / B}\right)\right)=1_{X / B}$ it can be rewritten as

$$
\mathfrak{c}\left(s_{\alpha}(u)\right) \cdot \mathfrak{c}\left(C_{\alpha}(1)\right)+\mathfrak{c}\left(\Delta_{-\alpha}(u)\right)=\mathfrak{c}\left(s_{\alpha}(u) C_{\alpha}(1)+\Delta_{-\alpha}(u)\right) .
$$

Using the definition of $C_{\alpha}$ and part (3) of Proposition 3.13 we finally obtain

$$
=\mathfrak{c}\left(s_{\alpha}(u) \cdot e_{\alpha}+\Delta_{-\alpha}(u)\right)=\mathfrak{c}\left(C_{-\alpha}\left(s_{-\alpha}(u)\right)\right)=\mathfrak{c}\left(C_{\alpha}(u)\right) .
$$

This proves the second formula.

The third formula follows from part (2) of Lemma 10.8 and from the fact that $C_{-\alpha} \Delta_{-\alpha}=0$ (see (6) of Prop. 3.13). The last formula is obtained by the composite of the second and the third one.

\section{Bott-Samelson varieties}

In the present section we compute an oriented cohomology of Bott-Samelson varieties. For definition and basic properties of Bott-Samelson varieties, we refer to papers [1], [19], [5] and [24].

11.1. - Let $G$ be a split semisimple linear algebraic group over a field $k$, let $T$ be a split maximal torus of $G$ and let $T \subset B$ be a Borel subgroup. Let $\left\{\alpha_{1}, \alpha_{2}, \ldots, \alpha_{n}\right\}$, where $n$ is the rank of $G$, be a set of simple roots of $G$. Let $P_{i}$ be a minimal parabolic subgroup corresponding to a simple root $\alpha_{i}$, i.e., the subgroup generated by unipotent subgroups $U_{\alpha_{j}}$, $1 \leq j \leq n$ and $U_{-\alpha_{i}}$.

Definition 11.2. - For an $l$-tuple of integers $I=\left(i_{1}, i_{2}, \ldots, i_{l}\right)$ with $1 \leq i_{j} \leq n$, we define a variety $X_{I}$ to be the fiber product

$$
X_{I}=P_{i_{1}} \times{ }^{B} P_{i_{2}} \times{ }^{B} \cdots \times{ }^{B} P_{i_{l}} .
$$

If $I=\varnothing$, then we set $X_{\varnothing}=p t$.

Observe that there is a natural right action of $B$ on $X_{I}$, the quotient $X_{I} / B$ exists and $X_{I} \rightarrow X_{I} / B$ is a principal $B$-bundle. The variety $X_{I} / B$ is called a Bott-Samelson variety corresponding to $I$.

11.3. - Let $J=\left(i_{1}, i_{2}, \ldots, i_{l-1}\right)$. Then $X_{I}=X_{J} \times{ }^{B} P_{i_{l}}$, and we are in the situation of 10.3 for $P=P_{i_{l}}, \alpha=\alpha_{i_{l}}, X^{\prime}=X_{I}$ and $X=X_{J}$. In particular, the projection map

$$
p_{I}: X_{I} / B \rightarrow X_{J} / B
$$

has a structure of a $\mathbb{P}^{1}$-bundle with a section denoted by $\sigma_{I}$.

Observe that the structure map $\pi_{I}: X_{I} / B \rightarrow$ pt can be written as a composite of projection maps

$$
X_{I} / B \rightarrow X_{\left(i_{1}, \ldots, i_{l-1}\right)} / B \rightarrow \cdots \rightarrow X_{\left(i_{1}\right)} / B \rightarrow \mathrm{pt}
$$

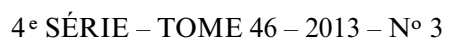


Hence, the variety $X_{I} / B$ is obtained by means of a sequence of split $\mathbb{P}^{1}$-bundles.

According to 10.1 for an oriented cohomology theory h with a formal group law $F$ there is a well-defined characteristic map

$$
\mathfrak{c}_{I}: R \llbracket M \rrbracket_{F} \rightarrow \mathrm{h}\left(X_{I} / B\right),
$$

where $R \llbracket M \rrbracket_{F}$ is the formal group ring of the group of characters $M$ of $T$. Observe that $\mathfrak{c}_{\varnothing}=\epsilon$ is the augmentation map. Applying Theorem 9.3 and Corollary 10.7 we obtain an isomorphism

$$
\mathrm{h}\left(X_{I} / B\right) \simeq \mathrm{h}(\mathrm{pt})\left[\xi_{1}, \xi_{2}, \ldots, \xi_{l}\right] /\left(\left\{\xi_{j}^{2}-y_{j} \xi_{j}\right\}_{j=1 \ldots l}\right)
$$

where $y_{j}=p^{*} \mathfrak{c}_{\left(i_{1}, \ldots, i_{j-1}\right)}\left(x_{-\alpha_{i_{j}}}\right)$ and $p^{*}$ denotes the pull-back to $\mathrm{h}\left(X_{I} / B\right)$.

Theorem 11.4. - Let $X_{I} / B$ be the Bott-Samelson variety corresponding to an l-tuple $I=\left(i_{1}, \ldots, i_{l}\right)$. For any subset $K$ of $[1, l]$ we define

$$
\xi_{K}=\prod_{j \in K} \xi_{j} \quad \text { and } \quad \Theta_{K}=\Theta_{1} \cdots \Theta_{l}, \text { where } \Theta_{j}= \begin{cases}\Delta_{-\alpha_{i_{j}}} & \text { if } j \in K, \\ s_{\alpha_{i_{j}}} & \text { otherwise. }\end{cases}
$$

Then the elements $\xi_{K}$, where $K$ runs through all subsets of $[1, l]$, form a basis of the free $\mathrm{h}(\mathrm{pt})$-module $\mathrm{h}\left(X_{I} / B\right)$. As a ring $\mathrm{h}\left(X_{I} / B\right)$ is a quotient of the polynomial ring $\mathrm{h}(\mathrm{pt})\left[\xi_{1}, \ldots, \xi_{l}\right]$ modulo the relations

$$
\xi_{j}^{2}=\sum_{K \subseteq[1, j-1]} \epsilon \Theta_{K}\left(x_{-\alpha_{i_{j}}}\right) \xi_{K} \xi_{j}
$$

Proof. - The fact that $\mathrm{h}\left(X_{I} / B\right)$ is a free $\mathrm{h}(\mathrm{pt})$-module with a basis $\xi_{K}$ follows by the projective bundle theorem, since $X_{I} / B$ is obtained by means of a sequence of split $\mathbb{P}^{1}$-bundles. To obtain the relations, we first use Lemma 10.8.(2) to prove by induction that

$$
\mathfrak{c}_{\left(i_{1}, \ldots, i_{j}\right)}(u)=\sum_{K \subseteq[1, j]} \epsilon \Theta_{K}(u) \xi_{K} .
$$

Using this we then compute $y_{j}$ and plug the result into the Formula (5).

The following lemma provides a formula for the push-forward of the structure map $\pi_{I}: X_{I} / B \rightarrow \mathrm{pt}:$

Lemma 11.5. - Consider the variety $X_{I} / B$ for a tuple $I=\left(i_{1}, \ldots, i_{l}\right)$. For any $u \in R \llbracket M \rrbracket_{F}$ we have

$$
\pi_{I *}\left(\mathfrak{c}_{I}(u)\right)=\epsilon C_{I}(u)
$$

where $C_{I}=C_{\alpha_{1}} \ldots C_{\alpha_{l}}$ is the composite of operators defined in 3.12.

Proof. - Decomposing the structure map as $\pi_{I}: X_{I} / B \stackrel{p_{I}}{\rightarrow} X_{J} / B \stackrel{\pi_{J}}{\rightarrow}$ pt, where $J=\left(i_{1}, \ldots, i_{l-1}\right)$ and applying Prop. 10.10.(2) we obtain

$$
\pi_{I *}\left(\mathfrak{c}_{I}(u)\right)=\pi_{J *} p_{I *}\left(\mathfrak{c}_{I}(u)\right)=\pi_{J *}\left(\mathfrak{c}_{J}\left(C_{\alpha_{l}}(u)\right)\right) .
$$

Repeating this recursively for the structure map $\pi_{J}: X_{J} / B \rightarrow$ pt and so on, we obtain the desired formula. 


\section{Bott-Samelson resolutions}

In the present section we discuss the relations between Bott-Samelson varieties and Schubert varieties on $G / B$.

12.1. - According to Bruhat decomposition the variety of complete flags $G / B$ is a finite disjoint union of affine spaces $G / B=\coprod_{w \in W} B w B / B$, where $W$ is the Weyl group of $G$. The closure of a cell $B w B / B$ is denoted by $X_{w}$ and is called a Schubert variety. Observe that $X_{w}$ is not smooth in general.

Let $w=s_{i_{1}} s_{i_{2}} \ldots s_{i_{l}}$ be a (reduced) decomposition into a product of simple reflections (here $s_{i}$ denotes $s_{\alpha_{i}}$ ) with $l=l(w)$ being the length of $w$. The multiplication map induces a morphism

$$
q_{I}: X_{I} / B \rightarrow G / B, \text { where } I=\left(i_{1}, i_{2}, \ldots, i_{l}\right) .
$$

By the results of [1, Ch. 3] (see also [19, §1.7] and [5, §4]) this map factors as $q_{I}: X_{I} / B \rightarrow X_{w} \hookrightarrow G / B$, where the first map is surjective birational and the second is a closed embedding. Furthermore, when $J$ is $I$ with the last entry removed, we have $q_{I} \circ \sigma_{I}=q_{J}$.

Lemma 12.2. - Consider the characteristic map $\mathfrak{c}_{G / B}: R \llbracket M \rrbracket_{F} \rightarrow \mathrm{h}(G / B)$. For any $u \in R \llbracket M \rrbracket_{F}$ we have

$$
q_{I}^{*}\left(\mathfrak{c}_{G / B}(u)\right)=\mathfrak{c}_{I}(u) .
$$

Proof. - By definition of the map $q_{I}$ and the bundle $\mathscr{L}(\lambda)$ we have $q_{I}^{*}(\mathcal{L}(\lambda))=\mathscr{L}_{I}(\lambda)$, where $\mathscr{L}(\lambda)$ (resp. $\mathscr{L}_{I}(\lambda)$ ) is the line bundle over $G / B$ (resp. over $X_{I} / B$ ) corresponding to a character $\lambda$. Hence, $q_{I}^{*}\left(\mathfrak{c}_{G / B}\left(x_{\lambda}\right)\right)=\mathfrak{c}_{I}\left(x_{\lambda}\right)$. For a general $u$ it follows by continuity.

Let $w_{0}$ be the element of maximal length in $W$ and let $X=X_{I_{0}} / B$ be the Bott-Samelson variety where $I_{0}$ is a reduced decomposition of $w_{0}$.

Lemma 12.3. - Assume that $\mathrm{h}$ is weakly birationally invariant. Then the pull-back $q_{I_{0}}^{*}$ is injective.

Proof. - Since our theory is weakly birationally invariant, the push-forward of the fundamental class $q_{I_{0} *}(1)$ is invertible. The lemma now follows by projection formula.

Theorem 12.4 (cf. [5, Prop. 3]). - Assume that $\mathrm{h}$ is weakly birationally invariant. Consider the quotient map $p_{i}: G / B \rightarrow G / P_{i}$, where $1 \leq i \leq n$. Then for any $u \in R \llbracket M \rrbracket_{F}$ we have the equality

$$
p_{i}^{*} p_{i *}\left(\mathfrak{c}_{G / B}(u)\right)=\mathfrak{c}_{G / B}\left(C_{\alpha_{i}}(u)\right) .
$$

Proof. - We choose a reduced decomposition $I_{0}$ of $w_{0}$ in simple reflections such that the last reflection is $s_{i}$. Let $J$ be the same tuple without the last entry. Consider the Cartesian diagram

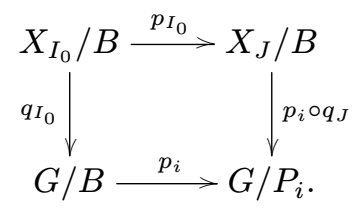

4 ${ }^{\text {e }}$ SÉRIE - TOME $46-2013$ - No 3 
Since the horizontal map $p_{i}$ is smooth, the diagram is transversal. Since $q_{I_{0}}^{*}$ is injective by Lemma 12.3, it is enough to prove the equality after applying $q_{I_{0}}^{*}$ to both sides. By base change and by transversality of the diagram we have

$$
q_{I_{0}}^{*} p_{i}^{*} p_{i *}\left(\mathfrak{c}_{G / B}(u)\right)=p_{I_{0}}^{*}\left(p_{i} \circ q_{J}\right)^{*} p_{i *}\left(\mathfrak{c}_{G / B}(u)\right)=p_{I_{0}}^{*} p_{I_{0} *} q_{I_{0}}^{*}\left(\mathfrak{c}_{G / B}(u)\right) .
$$

By Lemma 12.2 and by Prop. 10.10.(4) we obtain

$$
=p_{I_{0}}^{*} p_{I_{0} *}\left(\mathfrak{c}_{I_{0}}(u)\right)=\mathfrak{c}_{I_{0}}\left(C_{\alpha_{i}}(u)\right)=q_{I_{0}}^{*}\left(\mathfrak{c}_{G / B}\left(C_{\alpha_{i}}(u)\right)\right)
$$

and the theorem is proved.

\section{PART III ALGEBRAIC AND GEOMETRIC COMPARISON. APPLICATIONS}

\section{Comparison results}

In the present section we explain why the ring $\mathcal{H}(M)_{F}$ is naturally isomorphic to the ring $\mathrm{h}(G / B)$ (see 13.13) for most weakly birationally invariant theories.

13.1. - Let $I$ be a sequence of simple reflections. Recall that $w(I)=s_{i_{1}} \cdots s_{i_{l}}$ is the corresponding product of simple reflections. Let

$$
\zeta_{I}=\left(q_{I}\right)_{*}(1) \in \mathrm{h}(G / B)
$$

denote the push-forward of the fundamental class of the Bott-Samelson variety corresponding to $I$.

We consider the characteristic map $\mathfrak{c}_{G / B}: R \llbracket M \rrbracket_{F} \rightarrow \mathrm{h}(G / B)$. Let $u_{0} \in \mathcal{J}^{N}$ be chosen as in Section 5.

Lemma 13.2. - Let $I_{w}$ be a reduced decomposition of an element $w$. Then

$$
\zeta_{I_{w}} \cdot \mathfrak{c}_{G / B}\left(u_{0}\right)= \begin{cases}0 & \text { if } w \neq w_{0}, \\ \mathfrak{c}_{G / B}\left(u_{0}\right) & \text { if } w=w_{0} .\end{cases}
$$

Proof. - We have $\zeta_{I_{w}} \cdot \mathfrak{c}_{G / B}\left(u_{0}\right)=q_{I_{w} *}\left(\mathfrak{c}_{I_{w}}\left(u_{0}\right)\right)$ by the projection formula and the fact that $q_{I_{w}}^{*} \mathfrak{c}_{G / B}=\mathfrak{c}_{I_{w}}$. By Formula (6), $\mathfrak{c}_{I_{w}}\left(u_{0}\right)=0$ if $l\left(I_{w}\right)<N$ i.e., if $w \neq w_{0}$. This proves the first part of the formula. The other case will follow if we show that $\zeta_{I_{w_{0}}}=1-\sum_{w \neq w_{0}} r_{w} \zeta_{I_{w}}$ for some coefficients $r_{w}$ in $R$. For this, it suffices to show that the coefficient $r_{w_{0}}$ in front of $\zeta_{I_{w_{0}}}$ in the decomposition of 1 on the basis of the $\zeta_{I_{w}}$ is 1 . We have the commutative diagram

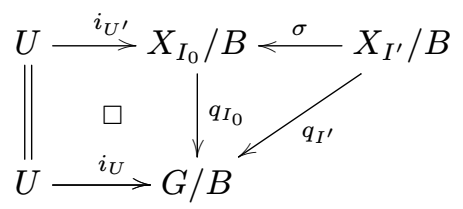

where $I_{0}$ is any reduced decomposition of $w_{0}, I^{\prime}$ is $I_{0}$ with its last entry removed, $\sigma$ is the section of $p_{I_{0}}, U$ is the big open cell in $G / B$ and $U^{\prime}$ is its pre-image in $X_{I_{0}} / B$. By $[8, \S 3.10$ 
and 3.11], $U^{\prime}$ is indeed isomorphic to $U$ and it is included in the complement of $\sigma X_{I^{\prime}} / B$. Thus, $i_{U^{\prime}}^{*} \sigma_{*}=0$, using that $X_{I_{0}} / B \rightarrow X_{I^{\prime}} / B$ is a projective bundle. By base change on the cartesian diagram, we obtain $i_{U}^{*} q_{I^{\prime} *}=0$ and $i_{U}^{*} q_{I_{0} *}(1)=1$. Since any $I_{w}$ can be completed to a decomposition of $w_{0}$, this proves that $i_{U}^{*}\left(\zeta_{I_{w}}\right)=0$ if $w \neq w_{0}$. Pulling back $1=\sum_{w} r_{w} \zeta_{I_{w}}$ by $i_{U}$, we get $1=r_{w_{0}}$ (by homotopy invariance, $\mathrm{h}(U) \simeq R$ ).

Lemma 13.3. - We have

$$
\mathfrak{t} \zeta_{\varnothing}=\mathfrak{c}_{G / B}\left(u_{0}\right) \quad \text { and } \quad \mathfrak{t} \zeta_{I}=\mathfrak{c}_{G / B}\left(C_{I^{\mathrm{rev}}}\left(u_{0}\right)\right) .
$$

Proof. - Let $I_{w_{0}}=\left(i_{1}, \ldots, i_{N}\right)$. With the notation of Theorem 11.4, we have

$$
\begin{aligned}
\mathfrak{t} \zeta_{\varnothing} & =\mathfrak{t} q_{\varnothing *}(1)=\mathfrak{t} q_{I_{w_{0}} *} \sigma_{I_{w_{0}} *} \cdots \sigma_{\left(i_{1}\right) *}(1)=q_{I_{w_{0}} *}\left(\mathfrak{t} \xi_{[1, N]}\right)=q_{I_{w_{0}} *}\left(\mathfrak{c}_{I_{w_{0}}}\left(u_{0}\right)\right) \\
& =q_{I_{w_{0}} *}(1) \mathfrak{c}_{G / B}\left(u_{0}\right)=\mathfrak{c}_{G / B}\left(u_{0}\right)
\end{aligned}
$$

where the two last equalities follow from the projection formula together with $q_{I_{w}}^{*} \mathfrak{c}_{G / B}=\mathfrak{c}_{I_{w}}$ and then Lemma 13.2. For the second formula, which coincides with the first one when $I$ is empty, we use induction on the length of $I$. If $I=\left(i_{1}, \ldots, i_{l}\right)$ and $J=\left(i_{1}, \ldots, i_{l-1}\right)$, then by base change via diagram (7) we obtain

$$
\mathfrak{t} \cdot \zeta_{I}=\mathfrak{t} \cdot q_{I *}(1)=\mathfrak{t} \cdot q_{I *} p_{I}^{*}(1)=\mathfrak{t} \cdot p_{i_{l}}^{*}\left(p_{i_{l}} \circ q_{J}\right)_{*}(1)=p_{i_{l}}^{*} p_{i_{l} *}\left(\mathfrak{t} \cdot \zeta_{J}\right) .
$$

By the induction step and Theorem 12.4 we obtain that this is equal to

$$
p_{i_{l}}^{*} p_{i_{l} *}\left(\mathfrak{c}_{G / B}\left(C_{J^{\mathrm{rev}}}\left(u_{0}\right)\right)\right)=\mathfrak{c}_{G / B}\left(C_{\alpha_{i_{l}}} \circ C_{J^{\mathrm{rev}}}\left(u_{0}\right)\right)=\mathfrak{c}_{G / B}\left(C_{I^{\mathrm{rev}}}\left(u_{0}\right)\right)
$$

and the proof is finished.

Lemma 13.4. - Let $\pi: G / B \rightarrow$ pt be the structure map. For any sequence $I$ and $u \in R \llbracket M \rrbracket_{F}$ we have

$$
\pi_{*}\left(\zeta_{I} \cdot \mathfrak{c}_{G / B}(u)\right)=\epsilon C_{I}(u)
$$

Proof. - The projection formula and Lemma 11.5 imply that we have

$$
\pi_{*}\left(\zeta_{I} \cdot \mathfrak{c}_{G / B}(u)\right)=\pi_{*}\left(q_{I *}(1) \cdot \mathfrak{c}_{G / B}(u)\right)=\pi_{*} q_{I *} q_{I}^{*}\left(\mathfrak{c}_{G / B}(u)\right)=\pi_{I *}\left(\mathfrak{c}_{I}(u)\right)=\epsilon C_{I}(u) .
$$

Let us now discuss the following assumption.

Assumption 13.5. - For each element $w \in W$, let $I_{w}$ be a chosen reduced decomposition of $w$. The elements $\zeta_{I_{w}}$, where $w$ runs through all elements of the Weyl group $W$, form an $R$-basis of the cohomology $\mathrm{h}(G / B)$.

Lemma 13.6. - If $\mathfrak{t}$ is regular in $R$, then the $\zeta_{I_{w}}$ are $R$-linearly independent.

Proof. - Assume that $\sum_{w} r_{w} \zeta_{I_{w}}=0$ for some $r_{w} \in R$. Then for any $I$,

$$
0=\pi_{*}\left(\left(\sum_{w} r_{w} \zeta_{I_{w}}\right) \mathfrak{c}_{G / B}\left(C_{I}\left(u_{0}\right)\right)\right)=\sum_{w} r_{w} \epsilon C_{I_{w}} C_{I}\left(u_{0}\right)
$$

by Lemma 13.4. Then, by part (3) of Lemma 5.3, the matrix $\left(\epsilon C_{I_{w}} C_{I_{v}}\left(u_{0}\right)\right)_{v, w \in W}$ has zero kernel, because it is "triangular" with the regular element $\mathfrak{t}$ on the diagonal.

Lemma 13.7. - Assumption 13.5 holds for

1. Chow groups (in arbitrary characteristic);

2. K-theory (in arbitrary characteristic); 
3. any oriented cohomology theory $\mathrm{h}_{1}^{*}=\mathrm{h}_{0}^{*} \otimes_{R_{0}} R_{1}$ obtained from another one $\mathrm{h}_{0}^{*}$ for which the assumption already holds;

4. any oriented cohomology theory that satisfies the weak localization axiom and such that $\mathfrak{t}$ is regular in $\mathrm{h}(\mathrm{pt})$;

5. any oriented cohomology theory such that $\mathrm{h} \otimes_{\mathrm{h}(\mathrm{pt})} \mathbb{Z}=\mathrm{CH}$, e.g., algebraic cobordism and connective $K$-theory.

Proof. - Part (3) is obvious since a free module stays free by base change. For Chow groups and K-theory, this is classical and for example proved in [8]. When $\mathrm{h}$ satisfies the weak localization axiom, by Theorem 8.8 applied to the filtration of $G / B$ by open subsets obtained by adding open Schubert cells one by one, by increasing codimension, one gets that the $\zeta_{I_{w}}$ generate $\mathrm{h}(G / B)$. When furthermore $\mathrm{t}$ is regular in $\mathrm{h}(\mathrm{pt})$, Lemma 13.6 shows that they are independent. For algebraic cobordism, since $G / B$ is a cellular space and classes of Schubert varieties form a $\mathbb{Z}$-basis of $\mathrm{CH}(G / B)$, the lemma follows from [23, Cor. 2.9].

Recall from Definition 6.1 that we also have an algebraic characteristic map $\mathfrak{c}: R \llbracket M \rrbracket_{F} \rightarrow \mathcal{H}(M)_{F}$.

Lemma 13.8. - Assume the torsion index $\mathfrak{t}$ is invertible in $R$ and that Assumption 13.5 is satisfied. Then, there is a unique morphism $\theta: \mathcal{H}(M)_{F} \rightarrow \mathrm{h}(G / B)$ of $R$-algebras such that $\mathfrak{c}_{G / B}=\theta \circ \mathfrak{c}$.

Proof. - We fix a reduced decomposition $I_{w}$ for each $w \in W$. By 6.4, the map $\mathfrak{c}$ is surjective. We can therefore find a pre-image $u_{w}$ of each element $z_{I_{w}}^{C}$ of Theorem 6.3. By the form of the characteristic map given there, these elements satisfy $\epsilon C_{I_{w}}\left(u_{v}\right)=\delta_{w, v}$. Let us set $\tau_{I_{w}}=\mathfrak{c}_{G / B}\left(u_{w}\right)$. By the previous lemma, $\left(\tau_{I_{w}}\right)_{w \in W}$ is a basis of $\mathrm{h}(G / B)$ that is dual basis to the basis $\left(\zeta_{I_{w}}\right)_{w \in W}$ with respect to the bilinear form $(\zeta, \tau) \mapsto \pi_{*}(\zeta . \tau)$, and this form is therefore nondegenerate. We now define $\theta$ as the $R$-linear morphism sending $z_{I_{w}}^{C}$ to $\tau_{I_{w}}$. By the formula $\pi_{*}\left(\zeta_{I_{w}} \cdot \mathfrak{c}_{G / B}(u)\right)=\epsilon C_{I_{w}}(u)$, we must have

$$
\mathfrak{c}_{G / B}(u)=\sum_{w \in W} \epsilon C_{I_{w}}(u) \tau_{I_{w}}
$$

and the isomorphism $\theta$ therefore satisfies $\mathfrak{c}_{G / B}=\theta \circ \mathfrak{c}$ since the $z_{I_{w}}^{C}$ satisfy the same formula with $\mathfrak{c}$. The uniqueness of $\theta$ is immediate by surjectivity of $\mathfrak{c}$.

COROllary 13.9. - When $\mathfrak{t}$ is invertible in $R$ and Assumption 13.5 holds, the characteristic map $\mathfrak{c}_{G / B}$ is surjective.

Proof. - Use Theorem 6.4 and the previous lemma.

COROLlary 13.10. - When $\mathfrak{t}$ is invertible in $R$ which has no 2-torsion and Assumption 13.5 holds, the kernel of the characteristic map $\mathfrak{c}_{G / B}$ is the ideal of $R \llbracket M \rrbracket_{F}$ generated by elements in I fixed by $W$.

Proof. - Use Theorem 6.9 and the previous lemma.

Lemma 13.11. - Under the assumptions of Proposition 13.8, we have $\zeta_{\varnothing}=\tau_{I_{w_{0}}}$. In particular, $\tau_{I_{w_{0}}}$ does not depend on the choices of the reduced decompositions $I_{w}$. 
Proof. - By definition of the dual basis $\left(\tau_{I_{w}}\right)_{w \in W}$, it suffices to show that we have $\pi_{*}\left(\zeta_{I_{w}} \cdot \zeta_{\varnothing}\right)=\delta_{w_{0}, w}$. By Lemmas 13.3 and 13.4, we have

$$
\pi_{*}\left(\zeta_{I_{w}} \cdot \zeta_{\varnothing}\right)=\pi_{*}\left(\zeta_{I_{w}} \cdot \mathfrak{t}^{-1} \mathfrak{c}_{G / B}\left(u_{0}\right)\right)=\mathfrak{t}^{-1} \epsilon C_{I_{w}}\left(u_{0}\right)=\delta_{w, w_{0}} .
$$

Let $\tilde{A}_{i}$ denote the operator $p_{i}^{*}\left(p_{i}\right)_{*}$ on $\mathrm{h}(G / B)$ and let $\tilde{A}_{I}$ be as usual for a sequence $I$.

Lemma 13.12. - The isomorphism $\theta$ of Lemma 13.8 satisfies $\theta A_{I}=\tilde{A}_{I} \theta$ and therefore sends the $A_{I_{w}}\left(z_{0}\right)$ to the $\tilde{A}_{I_{w}}\left(\zeta_{\varnothing}\right)$.

Proof. - It follows from the surjectivity of the characteristic map, the fact that $\theta \circ \mathfrak{c}=\mathfrak{c}_{G / B}$, Theorem 12.4 and Proposition 7.8 part (1).

We can now show that the isomorphism $\theta$ of Lemma 13.8 descends to the case where $t$ is regular but not necessarily invertible.

Theorem 13.13. - Let $\mathrm{h}$ be a weakly birationally invariant theory satisfying Assumption 13.5 and such that $\mathfrak{t}$ is regular in $R=\mathrm{h}(\mathrm{pt})$. Then there is a unique isomorphism of $R$-algebras $\theta: \mathcal{H}(M)_{F} \rightarrow \mathrm{h}(G / B)$ such that the characteristic map $\mathfrak{c}_{G / B}$ is given by $\theta \circ \mathfrak{c}$. It satisfies $\theta \circ A_{I}=\tilde{A}_{I} \circ \theta$.

Proof. - We know from 7.9 that the $A_{I_{w}}\left(z_{0}\right)$ are a basis of $\mathcal{H}(M)_{F}$ and from Assumption 13.5 that the $\zeta_{I_{w}}$ are a basis of $\mathrm{h}(G / B)$. We define $\theta$ as the isomorphism sending $A_{I_{w}}\left(z_{0}\right)$ to $\zeta_{I_{w}}$. By Lemma 13.12, this definition coincides with the one of Lemma 13.8 when $\mathfrak{t}$ is invertible in $R$. We therefore have a commutative diagram

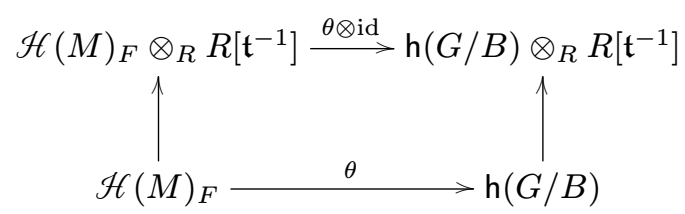

where $\theta \otimes$ id is the $\theta$ of Lemma 13.8 and the vertical maps are injective since $\mathcal{H}(M)_{F}$ and $\mathrm{h}(G / B)$ are free $R$-modules and $R$ injects in $R\left[\mathfrak{t}^{-1}\right]$. Since all other maps are ring morphisms, $\theta$ is one too, and the required equalities follow from the same ones in the case where $\mathfrak{t}$ is invertible. The morphism $\theta$ is unique since it is unique after inverting $\mathfrak{t}$.

REMARK 13.14. - Note that the morphism $\theta$ is independent of the choices of the $I_{w}$ used in its construction. This is obvious by surjectivity of the characteristic map after inverting $t$.

This completes the identification of $\mathcal{H}(M)_{F}$, the algebraic model for the cohomology ring introduced in Section 5, with the actual cohomology $\mathrm{h}(G / B)$.

4 e SÉRIE - TOME $46-2013$ - No 3 


\section{Formulas for push-forwards}

In the present section we assume that $\mathfrak{t}$ and 2 are regular in $R$. Note that given a formal group law $F$, both $R \llbracket M \rrbracket_{F}$ and $\mathcal{H}(M)_{F}$ can be defined without any reference to a cohomology theory. The goal of this section is to define an "algebraic push-forward" $\pi_{\mathscr{H}}: \mathcal{H}(M)_{F} \rightarrow R$ and to prove formulas related to it. Of course, when the formal group law comes from a cohomology theory, we prove that the morphism $\pi_{\mathscr{H}}$ corresponds to its geometric counterpart $\pi_{*}: \mathrm{h}(G / B) \rightarrow R$ through the isomorphism $\theta: \mathcal{H}(M)_{F} \stackrel{\simeq}{\rightarrow} \mathrm{h}(G / B)$ from Theorem 13.13. The general idea of the proofs is to use that there is a universal formal group law $U$ over the Lazard ring $\mathbb{L}$, and therefore that such formulas can be proved in $\mathscr{H}_{\mathbb{L}}(M)_{U}$, where they hold for geometric reasons, using algebraic cobordism. Then, they hold in any $\mathscr{H}(M)_{F}$ by specialization (even if it has no geometric origin).

14.1. - Recall that since $(U, \mathbb{L})$ is the universal formal group law, for any given formal group law $F$ over a ring $R$, there is a unique morphism $f: \mathbb{L} \rightarrow R$ sending $U$ to $F$. By Lemma 2.6 and Propositions 6.5 and 7.10, there is a commutative diagram

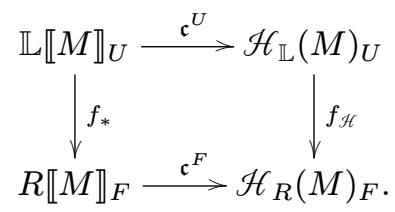

Furthermore, the morphism $f_{\mathscr{H}}$ commutes with the operators $A_{I}$ and $B_{I}$ and sends $z_{0}^{U}$ to $z_{0}^{R}$ by definition. Therefore, it sends $A_{I}^{U}\left(z_{0}^{U}\right)$ to $A_{I}^{F}\left(z_{0}^{R}\right)$ for any sequence $I$.

Let $\left(I_{w}\right)_{w \in W}$ be a choice of reduced decompositions. Then, by Prop. 7.9, the elements $\left(A_{I_{w}}\left(z_{0}\right)\right)_{w \in W}$ form an $R$-basis of $\mathcal{H}(M)_{F}$.

Definition 14.2. - Let $\pi_{\mathscr{H}}: \mathscr{H}(M)_{F} \rightarrow R$ be the $R$-linear morphism defined by $\pi_{\mathcal{H}}\left(A_{I_{w}}\left(z_{0}\right)\right)=\epsilon C_{I_{w}}(1)$.

Proposition 14.3. - We have

1. for any morphism $f: R \rightarrow R^{\prime}$ of rings sending a formal group law $F$ to $F^{\prime}$, we have $\pi_{\mathscr{H}}^{F}=\pi_{\mathscr{H}}^{F^{\prime}} \circ f_{\mathscr{H}}$.

2. Assume that $\mathrm{h}$ is an oriented cohomology theory satisfying the assumptions of Theorem 13.13, in which the isomorphism $\theta: \mathcal{H}(M)_{F} \cong \mathrm{h}(G / B)$ is defined. Then the morphism $\pi_{\mathscr{H}}$ satisfies $\pi_{\mathcal{H}} \circ \theta=\theta \circ \pi_{*}$. In other words, the morphism $\pi_{\mathscr{H}}$ is an algebraic replacement for the push-forward $\pi_{*}$.

Proof. - Since $f_{\mathcal{H}}\left(A_{I_{w}}^{F}\left(z_{0}^{F}\right)\right)=A_{I_{w}}^{F^{\prime}}\left(z_{0}^{F^{\prime}}\right)$, (1) follows from the definition of $\pi_{\mathscr{H}}$. Since the basis of $\mathrm{h}(G / B)$ formed by the $\zeta_{I_{w}}$ and the basis of $\mathcal{H}(M)_{F}$ formed by the $A_{I_{w}}\left(z_{0}\right)$ correspond to each other through the isomorphism $\theta$, (2) follows from Lemma 13.4.

COROLlaRY 14.4. - For any sequence I the morphism $\pi_{\mathcal{H}}$ of the proposition satisfies

$$
\pi_{\mathcal{H}}\left(A_{I^{\mathrm{rev}}}\left(z_{0}\right) \mathfrak{c}(u)\right)=\epsilon C_{I}(u) .
$$

In particular, it is independent of the choice $\left(I_{w}\right)_{w \in W}$ of reduced decompositions. 
Proof. - By Proposition 14.3 (2) and by Lemma 13.4, the formula holds for $\mathscr{H}_{\mathbb{L}}(M)_{U}$. In general, it reduces to the case where $\mathfrak{t}$ is invertible. When $v \in R \llbracket M \rrbracket_{F}^{W}$, we have $\epsilon C_{I}(v u)=\epsilon(v) \epsilon C_{I}(u)$ and $\mathfrak{c}(v u)=\epsilon(v) \mathfrak{c}(u)$ so by decomposing $u$ on the $C_{I_{w}}\left(u_{0}\right)$, which form an $R \llbracket M \rrbracket_{F}^{W}$-basis of $R \llbracket M \rrbracket_{F}$, it suffices to prove the formula for $u=C_{I_{w}}\left(u_{0}\right)$. For those, it follows by specialization using $f_{\mathscr{H}}$ from $\mathcal{H}_{\mathbb{L}}(M)_{U}$.

Remark 14.5. - Note that the elements $\pi_{\mathcal{H}}\left(A_{I^{\mathrm{rev}}}\left(z_{0}\right)\right)$ are particularly important because they represent the images of desingularized Schubert varieties in the cohomology of the point.

Proposition 14.6. - The morphism $\pi_{\mathscr{H}}$ satisfies

$$
\pi_{\mathcal{H}}\left(A_{I_{v}^{\mathrm{rev}}}\left(z_{0}\right) \cdot z_{I_{w}}^{C}\right)=\delta_{v, w} .
$$

In other words, the bilinear form $\pi_{\mathcal{H}}(-,-)$ is nondegenerate and the bases $\left(z_{I_{w}}^{C}\right)_{w \in W}$ and $\left(A_{I_{w}^{\mathrm{rev}}}\left(z_{0}\right)\right)_{w \in W}$ are dual to each other.

Proof. - The formula can be computed after extending scalars to $R\left[\mathfrak{t}^{-1}\right]$. Then, since the characteristic map $\mathfrak{c}$ is surjective, the elements $z_{I_{w}}^{C}$ have preimages $u_{w}$, such that $\epsilon C_{I_{v}}\left(u_{w}\right)=\delta_{v, w}$, by the expression of $\mathfrak{c}$ given in Theorem 6.3. The result then follows from Formula (14.4).

Let $I_{0}$ be a reduced decomposition of the longest element $w_{0}$. When $\mathfrak{t}$ is invertible, the element $y_{0}=C_{I_{0}^{\text {rev }}}\left(u_{0}\right) / \mathfrak{t}$ is invertible (by Lemma 5.3), and $\mathfrak{c}\left(y_{0}\right)=A_{I_{0}^{\mathrm{rev}}}\left(z_{0}\right)$ by Proposition 7.8 .

Lemma 14.7. - When $\mathfrak{t}$ is invertible in $R$, the morphism $\pi_{\mathcal{H}}$ satisfies

$$
\pi_{\mathcal{H}}(\mathfrak{c}(u))=\epsilon C_{I_{0}}\left(u y_{0}^{-1}\right) .
$$

Proof. - We have

$$
\pi_{\mathscr{H}}(\mathfrak{c}(u))=\pi_{\mathcal{H}}\left(\mathfrak{c}\left(u y_{0}^{-1}\right) \mathfrak{c}\left(y_{0}\right)\right)=\pi_{\mathcal{H}}\left(\mathfrak{c}\left(u y_{0}^{-1} A_{I_{0}^{\mathrm{rev}}}\left(u_{0}\right)\right)\right)=\epsilon C_{I_{0}}\left(u y_{0}^{-1}\right) .
$$

Proposition 14.8. - The operators $C$ satisfy the formula

$$
\epsilon C_{I}\left(u C_{J}\left(u_{0}\right)\right)=\epsilon C_{J^{\mathrm{rev}}}\left(u C_{I^{\mathrm{rev}}}\left(u_{0}\right)\right)
$$

for any pair of sequences $I$ and $J$. In particular $(u=1, J=\varnothing)$, for any sequence $I$, we have $\epsilon C_{I}\left(u_{0}\right)=\epsilon C_{I^{\mathrm{rev}}}\left(u_{0}\right)$.

Proof. - The formula can be proved after extension to $R\left[\mathfrak{t}^{-1}\right]$. It then follows from the computation of $\pi_{\mathscr{H}}$ in Formula (14.4) applied to both ends of

$$
A_{I^{\mathrm{rev}}}\left(z_{0}\right) \mathfrak{c}\left(u C_{J}\left(u_{0}\right)\right)=\mathfrak{t c}\left(C_{I^{\mathrm{rev}}}\left(u_{0}\right) u C_{J}\left(u_{0}\right)\right)=\mathfrak{c}\left(u C_{I^{\mathrm{rev}}}\left(u_{0}\right)\right) A_{J}\left(z_{0}\right) .
$$

REMARK 14.9. - The formulas given in this section are purely algebraic, but their proofs use geometric properties of cobordism. It would be interesting to have algebraic proofs derived directly from the various formulas in Section 3. 


\section{Algorithm for multiplying in $\mathrm{h}(G / B)$}

Fixing a choice of reduced decompositions $I_{w}$ for all $w \in W$, we define $\tau_{I_{w}}$ as $\theta\left(z_{I_{w}}^{C}\right)$.

Proposition 15.1. - Under the assumptions of Theorem 13.13, the bilinear form $(b, a) \mapsto$ $\pi_{*}(b \cdot a)$ is non degenerate on $\mathrm{h}(G / B)$ and for any choice of reduced decompositions $I_{w}$, the basis $\left(\zeta_{I_{w}}\right)$ and $\left(\tau_{I_{w}}\right)$ are dual to each other.

Proof. - The form can be computed after scalar extension to $R\left[\mathfrak{t}^{-1}\right]$, in which case it follows from the proof of Lemma 13.8.

Now the multiplication algorithm goes as follows: Substituting $u=C_{I_{w}^{\text {rev }}}\left(u_{0}\right)$ in (8) and using that $\mathfrak{t} \zeta_{I_{w}}=\mathfrak{c}_{G / B}\left(C_{I_{w}^{\text {rev }}}\left(u_{0}\right)\right)$ we obtain the transition matrix from the basis $\left(\zeta_{I_{w}}\right)$ to the basis $\left(\tau_{I_{w}}\right)$. Substituting $u=C_{I_{w}^{\mathrm{rev}}}\left(u_{0}\right) C_{I_{w^{\prime}}^{\mathrm{rev}}}\left(u_{0}\right)$ in (8) we obtain the decomposition of the product $\zeta_{I_{w}} \zeta_{I_{w}}$ on the basis $\left(\tau_{I_{w}}\right)$, and we rewrite it in terms of the $\zeta_{I_{w}}$ by using the transition matrix. This also explains how to decompose any $\zeta_{I}$ ( $I$ not necessarily reduced) on a given basis of $\zeta_{I_{w}}$. Again, it suffices to substitute $u=C_{I}\left(u_{0}\right)$ in (8) and use the transition matrix.

REMARK 15.2. - Note that this algorithm is an improvement over the one in [5] or [13] in the following sense. In both of these articles, it is explained how to decompose a product of two generators $\zeta_{I}$ and $\zeta_{J}$ as a linear combination of other such generators. But starting from a basis (i.e., the $\zeta_{I_{w}}$ for a choice of a reduced decomposition for each $w \in W$ ), it is not explained how to obtain a linear combination containing only generators $\zeta_{I}$ with $I$ among the $I_{w}$ and an algorithm for redecomposing any given $\zeta_{I}$ on a chosen basis is not given either. This is crucial to compute multiplication tables.

\section{Landweber-Novikov operations}

In this section we provide an algorithm for computing the Landweber-Novikov operations $S^{L N}$ on $\Omega^{*}(G / B)$.

16.1. - Let us recall briefly the definition of $S^{L N}$ (details can be found in [14, § 4.1.9]). Consider a graded polynomial ring $\mathbb{Z}[\mathbf{t}]=\mathbb{Z}\left[t_{1}, t_{2}, \ldots, t_{k}, \ldots\right]$ in infinite number of variables; for a multi-index $I=\left(i_{1}, i_{2}, \ldots, i_{k}\right)$ we set

$$
\mathbf{t}^{I}=t_{1}^{i_{1}} t_{2}^{i_{2}} \ldots t_{k}^{i_{k}}
$$

Let $\lambda_{(\mathbf{t})}$ denote the formal power series

$$
\lambda_{(\mathbf{t})}(x)=x+\sum_{i=1}^{\infty} t_{i} x^{i+1} .
$$

Consider a twisted theory $\tilde{\Omega}$ of $\Omega[\mathbf{t}]=\Omega \otimes_{\mathbb{Z}} \mathbb{Z}[\mathbf{t}]$ (see $[15, \S 4]$ ). By definition $\tilde{\Omega}(X)=\Omega(X)[\mathbf{t}]$ for any $X$, its Chern class is given by the formula

$$
c_{1}^{\tilde{\Omega}}(\mathscr{L})=\lambda_{(\mathbf{t})}\left(c_{1}^{\Omega}(\mathscr{L})\right),
$$

and its formal group law is given by

$$
F(x, y)=\lambda_{(\mathbf{t})}\left(U\left(\lambda_{(\mathbf{t})}^{-1}(x), \lambda_{(\mathbf{t})}^{-1}(y)\right)\right) .
$$


By the universality of $\Omega$ there is a natural transformation $\Omega \rightarrow \tilde{\Omega}$ given by

$$
a \mapsto \sum_{I} S_{I}^{L N}(a) t^{I}, \quad a \in \Omega(X)
$$

where the components $S_{I}^{L N}$ are called Landweber-Novikov operations on $\Omega(X)$.

16.2. - By functoriality of $R \llbracket M \rrbracket_{F}$ in $R$ (see 2.6) the map $\mathbb{L} \simeq \Omega(\mathrm{pt}) \rightarrow \tilde{\Omega}(\mathrm{pt}) \simeq \mathbb{L}[\mathbf{t}]$ induces a homomorphism

$$
\mathbb{L} \llbracket M \rrbracket_{U} \rightarrow \mathbb{L}[\mathbf{t}] \llbracket M \rrbracket_{F},
$$

while functoriality with respect to formal group laws induces a homomorphism

$$
\begin{aligned}
\mathbb{L}[\mathbf{t}] \llbracket M \rrbracket_{F} & \rightarrow \mathbb{L}[\mathbf{t}] \llbracket M \rrbracket_{U_{\Omega[\mathbf{t}]}} \\
x_{\mu} & \mapsto \lambda_{(\mathbf{t})}\left(x_{\mu}\right) .
\end{aligned}
$$

By (9) we have the following commutative diagram:

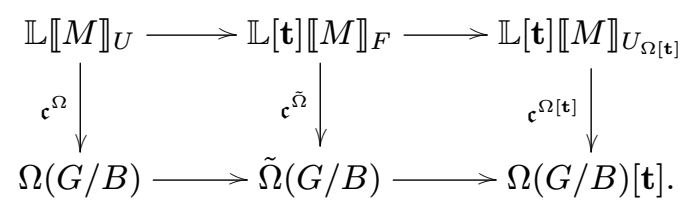

16.3. - An action of the Landweber-Novikov operation $S_{I}^{L N}$ on a basis element $\zeta_{I_{w}}$ can be computed as follows: First, we compute the image of $u=C_{I_{w}^{\text {rev }}}\left(u_{0}\right)$ under the composition of top horizontal arrows. Second, we extract the coefficient at the monomial $\mathbf{t}^{I}$ of this image. Finally, we apply the characteristic map $\mathrm{c}^{\Omega}$ to that coefficient. The result will give $S_{I}^{L N}\left(\zeta_{I_{w}}\right)$.

Indeed, by definition $S_{I}^{L N}\left(\zeta_{I_{w}}\right)$ is equal to the coefficient at $\mathbf{t}^{I}$ of the image of $\zeta_{I_{w}}$ under the composition of bottom horizontal arrows. Since $\mathfrak{t} \zeta_{I}=\mathfrak{c}_{G / B}\left(C_{I^{\mathrm{rev}}}\left(u_{0}\right)\right)$ and by commutativity of the diagram we are done.

\section{Examples of computations}

In the present section we list the multiplication tables for rings $\Omega^{*}(G / B)$, where $G$ has rank 2. The results are obtained by means of the algorithm described in Section 15 and realized in Macaulay 2 packages [6]. The answers for the other oriented cohomology theories are easily derived by a specialization of the coefficients of the universal formal group law. For instance, the answer for connective $K$-theory is obtained by specializing the coefficient $a_{1}$ to $v$ and all others to zero.

17.1. - We use the presentation of the Lazard ring

$$
\mathbb{L}=\mathbb{Z}\left[a_{1}, a_{2}, \ldots\right],
$$

$4^{\text {e }}$ SÉRIE - TOME $46-2013$ - No 3 
where the first generators $a_{i}$ are the following linear combinations of the coefficients $a_{i j}$ of the universal formal group law $U$ :

$$
\begin{aligned}
& a_{1}=a_{11} ; \\
& a_{2}=a_{12} ; \\
& a_{3}=a_{22}-a_{13} ; \\
& a_{4}=a_{14} ; \\
& a_{5}=-9 a_{15}+a_{24}+2 a_{33} .
\end{aligned}
$$

Note that the $a_{i}$ (resp. the $a_{i j}$ ) are of cohomological degree $-i$ (resp. $a_{1-i-j}$ ). For root systems of rank 2 , the longest element is of length at most $N=6$ (in the $G_{2}$ case), and we therefore need to compute the universal formal group law up to order 7 , which thus only involves $a_{1}, \ldots, a_{6}$. In fact, $a_{6}$ does not appear in the formulas: it is not difficult to show that $a_{N}$ will not appear in the multiplication formulas for a root system with longest element of length $N$.

17.2. - We use the upper case letter $\zeta_{I}$ for the element $\zeta_{I}=q_{I *}(1)$. For brevity $\zeta_{I}$ with $I=\left(i_{1}, \ldots, i_{l}\right)$ is denoted just by $\zeta_{i_{1} \ldots i_{l}}$, and when $I$ is the empty sequence, $\zeta_{I}$ is denoted by pt. Note that when $l(w)+l\left(w^{\prime}\right) \leq N=\operatorname{dim} G / B$ one has

$$
\zeta_{I_{w}} \zeta_{I_{w^{\prime}}}=\delta_{w, w_{0} w^{\prime}} \mathrm{pt}
$$

so we list the remaining cases only.

17.3. $-\left[A_{2}\right.$ case $]$

$$
\begin{aligned}
\zeta_{121} & =1+a_{2} \zeta_{1} \\
\zeta_{12}^{2} & =\zeta_{2} ; \\
\zeta_{21}^{2} & =\zeta_{1} \\
\zeta_{12} \zeta_{21} & =\zeta_{1}+\zeta_{2}+a_{1} \text { pt. }
\end{aligned}
$$

This agrees with the computations of Hornbostel-Kiritchenko in [13].

17.4. $-\left[B_{2}\right.$ case $]$

$$
\begin{aligned}
\zeta_{1212} & =1+2 a_{2} \zeta_{12}+\left(a_{3}-a_{1} a_{2}\right) \zeta_{2} \\
\zeta_{121}^{2} & =\zeta_{21} \\
\zeta_{212}^{2} & =2 \zeta_{12}+a_{1} \zeta_{2} \\
\zeta_{121} \zeta_{212} & =\zeta_{12}+\zeta_{21}+a_{1} \zeta_{1}+a_{1} \zeta_{2}+\left(2 a_{2}+a_{1}^{2}\right) \mathrm{pt} \\
\zeta_{121} \zeta_{12} & =\zeta_{1}+\zeta_{2}+a_{1} \mathrm{pt} \\
\zeta_{121} \zeta_{21} & =\zeta_{1} \\
\zeta_{212} \zeta_{12} & =\zeta_{2} ; \\
\zeta_{212} \zeta_{21} & =2 \zeta_{1}+\zeta_{2}+2 a_{1} \mathrm{pt} .
\end{aligned}
$$


17.5. $-\left[G_{2}\right.$ case $]$

$$
\begin{aligned}
& \zeta_{121212}=1+4 a_{2} \zeta_{1212}+\left(10 a_{3}-10 a_{1} a_{2}\right) \zeta_{212} \\
& -\left(4 a_{4}+9 a_{1} a_{3}+3 a_{2}^{2}-9 a_{1}^{2} a_{2}\right) \zeta_{12} \\
& -\left(54 a_{5}-459 a_{1} a_{4}-1188 a_{2} a_{3}-108 a_{1}^{2} a_{3}+1080 a_{1} a_{2}^{2}+108 a_{1}^{3} a_{2}\right) \zeta_{2} ; \\
& \zeta_{12121}^{2}=3 \zeta_{2121}+3 a_{1} \zeta_{121}+\left(13 a_{2}+2 a_{1}^{2}\right) \zeta_{21}+\left(2 a_{3}+7 a_{1} a_{2}+a_{1}^{3}\right) \zeta_{1} ; \\
& \zeta_{21212}^{2}=\zeta_{1212}+5 a_{2} \zeta_{12}+\left(6 a_{3}-5 a_{1} a_{2}\right) \zeta_{2} ; \\
& \zeta_{12121} \zeta_{21212}=\zeta_{1212}+\zeta_{2121}+a_{1} \zeta_{121}+a_{1} \zeta_{212}+\left(8 a_{2}+a_{1}^{2}\right) \zeta_{12}+\left(8 a_{2}+a_{1}^{2}\right) \zeta_{21} \\
& +\left(4 a_{3}+8 a_{1} a_{2}+a_{1}^{3}\right) \zeta_{1}+\left(10 a_{3}+6 a_{1} a_{2}+a_{1}^{3}\right) \zeta_{2} \\
& +\left(-4 a_{4}+a_{1} a_{3}+13 a_{2}^{2}+15 a_{1}^{2} a_{2}+a_{1}^{4}\right) \mathrm{pt} \\
& \zeta_{12121} \zeta_{1212}=\zeta_{121}+3 \zeta_{212}+4 a_{1} \zeta_{12}+3 a_{1} \zeta_{21}+\left(8 a_{2}+4 a_{1}^{2}\right) \zeta_{1}+\left(13 a_{2}+5 a_{1}^{2}\right) \zeta_{2} \\
& +\left(a_{3}+16 a_{1} a_{2}+5 a_{1}^{3}\right) \mathrm{pt} \\
& \zeta_{12121} \zeta_{2121}=2 \zeta_{121}+2 a_{1} \zeta_{21}+\left(4 a_{2}+a_{1}^{2}\right) \zeta_{1} \\
& \zeta_{21212} \zeta_{1212}=2 \zeta_{212}+a_{1} \zeta_{12}+4 a_{2} \zeta_{2} \\
& \zeta_{21212} \zeta_{2121}=\zeta_{121}+\zeta_{212}+a_{1} \zeta_{12}+a_{1} \zeta_{21}+\left(5 a_{2}+a_{1}^{2}\right) \zeta_{1}+\left(8 a_{2}+a_{1}^{2}\right) \zeta_{2} \\
& +\left(3 a_{3}+6 a_{1} a_{2}+a_{1}^{3}\right) \mathrm{pt} ; \\
& \zeta_{12121} \zeta_{121}=3 \zeta_{21}+2 a_{1} \zeta_{1} \\
& \zeta_{12121} \zeta_{212}=2 \zeta_{12}+\zeta_{21}+2 a_{1} \zeta_{1}+3 a_{1} \zeta_{2}+\left(4 a_{2}+3 a_{1}^{2}\right) \mathrm{pt} ; \\
& \zeta_{21212} \zeta_{121}=\zeta_{12}+2 \zeta_{21}+2 a_{1} \zeta_{1}+2 a_{1} \zeta_{2}+\left(4 a_{2}+2 a_{1}^{2}\right) \mathrm{pt} ; \\
& \zeta_{21212} \zeta_{212}=\zeta_{12} \\
& \zeta_{1212}^{2}=2 \zeta_{12}+a_{1} \zeta_{2} \\
& \zeta_{2121}^{2}=2 \zeta_{21}+a_{1} \zeta_{1} \\
& \zeta_{1212} \zeta_{2121}=2 \zeta_{12}+2 \zeta_{21}+3 a_{1} \zeta_{1}+4 a_{1} \zeta_{2}+\left(4 a_{2}+4 a_{1}^{2}\right) \mathrm{pt} ; \\
& \zeta_{12121} \zeta_{12}=\zeta_{1}+3 \zeta_{2}+3 a_{1} \mathrm{pt} \\
& \zeta_{12121} \zeta_{21}=\zeta_{1} \\
& \zeta_{21212} \zeta_{12}=\zeta_{2} \\
& \zeta_{21212} \zeta_{21}=\zeta_{1}+\zeta_{2}+a_{1} \mathrm{pt} \\
& \zeta_{1212} \zeta_{121}=2 \zeta_{1}+3 \zeta_{2}+4 a_{1} \mathrm{pt} \\
& \zeta_{1212} \zeta_{212}=\zeta_{2} \text {; } \\
& \zeta_{2121} \zeta_{121}=\zeta_{1} \\
& \zeta_{2121} \zeta_{212}=\zeta_{1}+2 \zeta_{2}+2 a_{1} \mathrm{pt} \text {. }
\end{aligned}
$$




\section{REFERENCES}

[1] R. Bott, H. Samelson, Applications of the theory of Morse to symmetric spaces, Amer. J. Math. 80 (1958), 964-1029.

[2] N. Bourbaki, Éléments de mathématique. Algèbre. Chapitres 1 à 3, Hermann, 1970; réédition Springer, 2007.

[3] N. Bourbaki, Éléments de mathématique. Algèbre commutative. Chapitres 1 à 4, Masson, 1985; réédition Springer, 2006.

[4] P. Bressler, S. Evens, The Schubert calculus, braid relations, and generalized cohomology, Trans. Amer. Math. Soc. 317 (1990), 799-811.

[5] P. Bressler, S. Evens, Schubert calculus in complex cobordism, Trans. Amer. Math. Soc. 331 (1992), 799-813.

[6] B. Calmès, V. Petrov, Cohomology of Borel varieties, a Macaulay 2 package, http: //www.math.uni-bielefeld.de/ bcalmes/M2packages/cohbovar.html, 2009.

[7] M. Demazure, Invariants symétriques entiers des groupes de Weyl et torsion, Invent. Math. 21 (1973), 287-301.

[8] M. Demazure, Désingularisation des variétés de Schubert généralisées, Ann. Sci. École Norm. Sup. 7 (1974), 53-88.

[9] W. Fulton, Intersection theory, second ed., Ergebn. Math. Grenzg. 2, Springer, 1998.

[10] D. R. Grayson, M. E. Stillman, Macaulay 2, a software system for research in algebraic geometry, http://www . math.uiuc.edu/Macaulay2/.

[11] R. Hartshorne, Algebraic geometry, Grad. Texts in Math. 52, Springer, 1977.

[12] M. J. Hopkins, N. J. Kuhn, D. C. Ravenel, Generalized group characters and complex oriented cohomology theories, J. Amer. Math. Soc. 13 (2000), 553-594.

[13] J. Hornbostel, V. Kiritchenko, Schubert calculus for algebraic cobordism, J. reine angew. Math. 656 (2011), 59-85.

[14] M. Levine, F. Morel, Algebraic cobordism, Springer Monographs in Math., Springer, 2007.

[15] A. Merkurjev, Algebraic oriented cohomology theories, in Algebraic number theory and algebraic geometry, Contemp. Math. 300, Amer. Math. Soc., 2002, 171-193.

[16] I. PAnin, Oriented cohomology theories of algebraic varieties, $K$-Theory 30 (2003), 265-314.

[17] I. PAnIN, Oriented cohomology theories of algebraic varieties. II (After I. Panin and A. Smirnov), Homology, Homotopy Appl. 11 (2009), 349-405.

[18] A. Preygel, Algebraic cobordism of varieties with G-bundles, preprint arXiv: 1007.0224.

[19] T. A. Springer, Schubert varieties and generalizations, in Representation theories and algebraic geometry (Montreal, PQ, 1997), NATO Adv. Sci. Inst. Ser. C Math. Phys. Sci. 514, Kluwer Acad. Publ., 1998, 413-440.

[20] B. Totaro, The torsion index of $E_{8}$ and other groups, Duke Math. J. 129 (2005), 219248.

[21] B. Totaro, The torsion index of the spin groups, Duke Math. J. 129 (2005), 249-290. 
[22] A. Vishik, Symmetric operations in algebraic cobordism, Adv. Math. 213 (2007), 489552.

[23] A. Vishik, N. Yagita, Algebraic cobordisms of a Pfister quadric, J. Lond. Math. Soc. 76 (2007), 586-604.

[24] M. Willems, Cohomologie équivariante des tours de Bott et calcul de Schubert équivariant, J. Inst. Math. Jussieu 5 (2006), 125-159.

Baptiste CALMÈs
Université d'Artois
Laboratoire de Mathématiques de Lens
Faculté des Sciences Jean Perrin
Rue Jean Souvraz, SP 18
62307 Lens Cedex, France
E-mail: baptiste.calmes@univ-artois.fr
Viktor PeTRov
27, Fontanka
191023 St. Petersburg, Russia
E-mail: victorapetrov@googlemail.com
Kirill ZAINOULLINE
University of Ottawa
585 King Edward
Ottawa ON K1N 6N5, Canada
E-mail: kirill@uottawa.ca

